\title{
Untargeted metabolic footprinting reveals key differences between fermented brown milk and fermented milk metabolomes
}

\author{
Jiangying Peng, ${ }^{1,2 *}$ (ㄴ) Liqing Ma, ${ }^{1,2 *}$ Lai-Yu Kwok, ${ }^{1,2} \odot$ Wenyi Zhang, ${ }^{1,2} \oplus$ and Tiansong Sun ${ }^{1,2} \dagger$ \\ ${ }^{1}$ Key Laboratory of Dairy Biotechnology and Engineering, Key Laboratory of Dairy Products Processing, Inner Mongolia Agricultural University, \\ Hohhot 010018, P. R. China \\ ${ }^{2}$ Inner Mongolia Key Laboratory of Dairy Biotechnology and Engineering, Inner Mongolia Agricultural University, Hohhot 010018, P. R. China
}

\begin{abstract}
Fermented brown milk has gained popularity because of its unique taste and flavor. Lactobacillus bulgaricus ND02 is a starter culture that has good milk fermentation characteristics. This study aimed to profile the metabolites produced during Maillard browning and to identify metabolomic differences between fermented brown milk and fermented milk produced by the ND02 strain. This study used liquid chromatography-mass spectrometry to compare the metabolomes of milk, fermented milk, brown milk, and fermented brown milk. Significant differences were observed in the abundances of various groups of metabolites, including peptides, AA, aldehydes, ketones, organic acids, vitamins, and nucleosides. The Maillard browning reaction significantly increased the intensity of a wide spectrum of flavor compounds, including short peptides, organic acids, and compounds of aldehydes, ketones, sulfur, and furan, which might together contribute to the unique flavor of brown milk. However, Maillard browning led to an increase in $\mathrm{N} \varepsilon$-(carboxymethyl)lysine, which might cause negative health effects such as diabetes, uremia, or Alzheimer's disease. On the other hand, fermenting brown milk with the ND02 strain effectively countered such an effect. Finally, 5 differentially abundant metabolites were identified between fermented brown milk and fermented milk, including L-lysine, methylglyoxal, glyoxal, 2,3-pentanedione, and 3-hydroxybutanoic acid, which might together contribute to the different nutritional qualities of fermented brown milk and fermented milk. This study has provided novel information about the Maillard reaction and compared the metabolomes of the 4 types of dairy products.
\end{abstract}

Key words: fermented brown milk, Maillard reaction, metabolomics, Lactobacillus bulgaricus ND02

Received June 9, 2021.

Accepted November 10, 2021.

*These authors contributed equally to this work.

†Corresponding author: sts9940@sina.com

\section{INTRODUCTION}

For many years, certain foods have been found to be beneficial to health. Fermented milk is a classic example, as it contains a wide range of bioactivities (e.g., modulating digestive and gastrointestinal functions, lowering cholesterol, and the capacity of immunoregulation; Buttriss, 1997). In terms of nutritional composition, fermented milk is an excellent source of high-quality protein, calcium, phosphorus, magnesium, zinc, niacin, vitamin $\mathrm{B} 12$, and riboflavin. Lactobacillus fermentation decreases the content of milk lactose, subsequently produces lactic acid production, and curdles the milk protein. These chemical changes offer the unique organoleptic characteristics and nutritional value to fermented dairy products. The main group of bacteria responsible for milk fermentation is lactic acid bacteria (LAB), and the fermentation process involves the following: (1) the formation of a large amount of lactic acid from lactose reduction; (2) an increase in free biomolecules, such as free AA, small peptides, and fatty acids; and (3) the production of flavor compounds from the metabolisms of carbohydrates and citrate, $\mathrm{AA}$, and fatty acids.

The development of cold chain technology has brought about new varieties of yogurt products. Brown yogurt, also known as char-grilled yogurt, is a new type of yogurt product. Instead of fresh milk, the milk protein and reducing sugar undergo the Maillard reaction at high temperature for a long time before fermentation by the starter culture. Fermented brown milk not only has a unique color and flavor as a result of the Maillard reaction (Han et al., 2019) but is beneficial to human health (Shida et al., 2017) due to the Lactobacillus fermentation products. Hence, fermented brown milk has become popular among consumers, particularly in China, Japan, Southeast Asia, Europe, and other regions (Xu et al., 2010). However, the complex Maillard reaction has been reported to produce a variety of potentially harmful compounds (Rannou et al., 2016). Therefore, detailed investigation into fermented brown milk metabolomes would be of great importance for 
assessing the health impact of consuming such products and ensuring product quality.

Metabolomics is a newly developed discipline after genomics and proteomics, and it provides an effective research platform for studying cell metabolites and building metabolic pathways (Rochfort, 2005). Metabolomic studies analyze low-molecular-weight substances (molecular weight $<1,000$ ) of a certain organism or subject under certain physiological conditions. These small compounds usually include short peptides, AA, nucleic acids, sugars, organic acids, vitamins, polyphenols, and other compounds such as alkaloids. Metabolomics can be divided into nontargeted metabolomics and targeted metabolomics based on research characteristics (Roberts et al., 2012). Targeted metabolomic analysis aims to quantify specific metabolites in environmental samples by building calibration curves of authentic standards (Roberts et al., 2012). Nontargeted metabolomic studies mainly detect dynamic changes of a large number of metabolites before and after an environmental disturbance or stimulation. Commonly used methods in metabolomics include GC-MS, nuclear magnetic resonance, and liquid chromatography (LC)MS. Among them, LC-MS combines the advantages of chromatography and MS that exhibits high separation ability for complex samples with a high selectivity and sensitivity, together with a powerful capacity in acquiring relative molecular mass and structural information. Metabolomic analyses are widely applied in many fields (e.g., medical studies, environmental, and food analysis).

Lactobacillus delbrueckii ssp. bulgaricus is the most common species used as an industrial dairy starter. The L. bulgaricus species has strong proteolytic ability, as its genome contains a cell wall proteolytic enzyme that can degrade $\mathrm{CN}$. The bacteria hydrolyze $\mathrm{CN}$ to produce all AA except glutamic acid and cysteine. In particular, L. bulgaricus can produce large amounts of proline, lysine, and valine (Do Carmo et al., 2011). In addition, L. bulgaricus has a complex lactose-degrading enzyme system that can efficiently convert lactose to lactic acid (Burgos-Rubio et al., 2000). The L. bulgaricus strain ND02 was isolated from traditional fermented yak milk in Qinghai, China (Sun et al., 2010). This strain possesses excellent fermentation properties in terms of time, flavor, and water-holding capacity of the produced fermented milk (Sun et al., 2010). The genome of ND02 contains 431 unique genes, including acetyltransferase, glutamate decarboxylase, polysaccharide synthesis protein, multiple glycosyltransferases, and extracellular polysaccharide synthesis cluster. The possession of these genes gives ND02 excellent curd characteristics and ability to produce fermented prod- ucts of good nutritional value. To gain a comprehensive understanding of the fermentation properties of ND02, it is necessary to characterize the metabolome of the resultant fermented milk products.

To the best of our knowledge, only a few studies have analyzed the metabolomic dynamics of brown milk fermentation, and no study has yet directly compared the metabolomes of fermented brown milk and fermented milk. Therefore, detailed investigation into the metabolites in fermented brown milk, especially Maillard reaction products, is essential for understanding the nutritional value of these products and to further improve the product quality. The aims of this work were to (1) identify and explore the role of the metabolites produced during Maillard browning of milk; (2) identify the metabolomic differences between fermented brown milk and fermented milk; and (3) to gain an in-depth understanding of the nutritional values of the 2 products.

\section{MATERIALS AND METHODS}

\section{Materials and Equipment}

The L. bulgaricus ND02 strain used in the experiment was obtained from the LAB Culture Collection, Inner Mongolia Agricultural University, China. Skim milk powder (protein 30\%) was purchased from Fonterra Co. Ltd. (NZMP Ltd.). The Acquity UPLC-Xevo-G2QTOF-MS system, MassLynx 4.1 Workstation, Progenesis QI, and the leucine-enkephalin standard were purchased from Waters Corp. Chromatographic grade acetonitrile, ammonium hydroxide solution, and formic acid were purchased from Sigma Aldrich.

\section{Sample Preparation}

Preparation of Milk and Fermented Milk. The reconstituted milk was prepared by suspending $10 \%$ (wt/wt) skim milk powder in water and then homogenizing it $\left(65^{\circ} \mathrm{C}, 90 \mathrm{MPa}\right)$ using a high-pressure homogenizer (Hirayamaha-300M). The mixture was sterilized at $95^{\circ} \mathrm{C}$ and maintained for $10 \mathrm{~min}$, followed by cooling to $42^{\circ} \mathrm{C}$. Then, L. bulgaricus ND02 $\left(5 \times 10^{6} \mathrm{cfu} / \mathrm{mL}\right)$ was inoculated into the sterilized milk, and the mixture was fermented at $42^{\circ} \mathrm{C}$ until the $\mathrm{pH}$ reached 4.5 . The whole process used anaerobic fermentation. Milk samples were collected after reaching the fermentation end point, and each sample was processed in sextuplicate. All samples were stored at $-80^{\circ} \mathrm{C}$ before analysis.

Preparation of Brown Milk and Fermented Brown Milk. Brown milk and fermented brown milk were prepared and sampled in the same way as the milk 
and fermented milk except that they were incubated in a thermostatically controlled water bath for $3 \mathrm{~h}$ for browning to occur after heating to $95^{\circ} \mathrm{C}$ instead of directly cooled to $42^{\circ} \mathrm{C}$.

\section{Acidity and Viable Counts}

The fermentation process was monitored by measuring the acidity of the fermented milk and the fermented brown milk. The $\mathrm{pH}$ value was determined by a $\mathrm{pH}$ meter (Leici). Titratable acidity (TA) was measured as described by Zha et al. (2021). Viable cell counts of L. bulgaricus ND02 were evaluated using the standard dilution method in de Man, Rogosa, and Sharpe (MRS) agar medium (Dan et al., 2019).

\section{Ultra Performance Liquid Chromatography- Quadrupole Time-of-Flight MS with $M S^{E}$ Data Acquisition}

Sample Pretreatment and Application. One milliliter of the sample was pipetted and mixed with 1 $\mathrm{mL}$ of acetonitrile solution in a 5 - $\mathrm{mL}$ Eppendorf tube for $1 \mathrm{~min}$. Afterward, the samples were centrifuged at $10,000 \times g$ at $4^{\circ} \mathrm{C}$ for $10 \mathrm{~min}$ to remove proteins. The supernatant $(1 \mathrm{~mL})$ was added into a new $5-\mathrm{mL}$ Eppendorf tube which contained $3 \mathrm{~mL}$ of acetonitrile solution, and the solution was allowed to stand at $4^{\circ} \mathrm{C}$ for $2 \mathrm{~h}$. The mixture was concentrated for $9 \mathrm{~h}$ using a vacuum rotary evaporation and then reconstituted by $40 \%$ acetonitrile solution. Reconstituted samples were filtered through a $0.22-\mu \mathrm{m}$ microporous membrane. A quality control (QC) sample was prepared by combining equal portion of all samples. Acetonitrile was used as a blank. Blank and QC samples were injected to the chromatographic system initially to ensure the stability of system and conditions. A total of 24 samples ( 6 each of milk, fermented milk, brown milk, and fermented brown milk) were injected randomly to minimize the effect of instrumental drift. A QC sample was injected every 3 samples and a standard QC to evaluate the mass accuracy.

Liquid Chromatography Conditions. Chromatographic analysis was performed using Waters Acquity ultra performance liquid chromatography (UPLC) system. The chromatographic column, HSS T3 (1.8 $\mu \mathrm{m}, 2.1 \mathrm{~mm} \times 100 \mathrm{~mm}$ ), was used for the separation. The column was maintained at $45^{\circ} \mathrm{C}$ during analysis. The injection volume was $5 \mu \mathrm{L}$, and the flow rate was $0.45 \mathrm{~mL} / \mathrm{min}$. The positive ion mode (electrospray ionization-positive, $\mathbf{E S I}^{+}$) mobile phase was made of $0.1 \%$ formic acid aqueous solution (A1) and $0.1 \%$ formic acid acetonitrile solution (B1). The negative ion mode $\left(\mathbf{E S I}^{-}\right)$mobile phase was made of $0.1 \%$ ammonium hydroxide (A2) and acetonitrile solution (B2). The gradient elution program used in both the $\mathrm{ESI}^{+}$ and $\mathrm{ESI}^{-}$modes was as follows: 0.25 min, $98 \% \mathrm{~A}, 2 \%$ B; 12 min, $2 \%$ A, $98 \%$ B; 14 min, $2 \%$ A, $98 \%$ B; 14.10 min, $98 \%$ A, $2 \%$ B; 17 min, $98 \%$ A, $2 \%$ B. The total run time was $17 \mathrm{~min}$.

Mass Spectrometry Conditions. Metabolites analysis of fermented milk and fermented brown milk was performed using UPLC-quadrupole-time-of-flight (UPLC-Q-TOF MS ${ }^{\mathrm{E}}$ ). Mass spectrometry was performed using $\mathrm{ESI}^{+}$and $\mathrm{ESI}^{-}\left(\mathrm{MS}^{\mathrm{E}}\right)$ Highly purified nitrogen and helium were used as the desolvation gas and collision gas, respectively. The data acquisition range was 50 to $1,200 \mathrm{~m} / z$. The collision energy of 20 $\mathrm{eV}$ to $40 \mathrm{eV}$ was applied to crush the parent ions. For both $\mathrm{ESI}^{+}$and $\mathrm{ESI}^{-}$modes, the source parameters were set as follows: the capillary voltage was $3.0 \mathrm{KV}$; the sample cone voltage was $40 \mathrm{~V}$; the source temperature was $120^{\circ} \mathrm{C}$; the desolvation temperature was $500^{\circ} \mathrm{C}$; the desolvation gas flow was $800 \mathrm{~L} / \mathrm{h}$; the cone gas flow was $50 \mathrm{~L} / \mathrm{h}$; and the scan time was $0.2 \mathrm{~s}$. A lock mass of leucine-enkephalin (concentration: $200 \mathrm{pmol}$ ) was used as the calibration solution $\left(\mathrm{ESI}^{+}: \mathrm{m} / z 556.2771\right.$; $\mathrm{ESI}^{-}$: $m / z 554.2615)$ and was introduced by Lockspray at 10 $\mu \mathrm{L} / \mathrm{min}$. The scan time was $0.2 \mathrm{~s}$, and the acquisition interval was $20 \mathrm{~s}$ for real-time data calibration.

Data Treatment and Metabolite Identification. The $\mathrm{MS}^{\mathrm{E}}$ raw data, including mass-to-charge ratio $(\mathrm{m} / \mathrm{z})$, retention time, and ionic strength, were collected by MassLynx 4.1 workstation. The $\mathrm{MS}^{\mathrm{E}}$ data were imported to the Progenesis QI software without any conversion to complete peak extraction, peak alignment, deconvolution, and normalization. According to standard ionization behavior, the adduct ions selected for data collection in the $\mathrm{ESI}^{+}$mode included $[\mathrm{M}+\mathrm{H}]^{+}$, $[\mathrm{M}+\mathrm{Na}]^{+}, \quad[\mathrm{M}+\mathrm{NH} 4]^{+},[\mathrm{M}+\mathrm{K}]^{+}$, and $[2 \mathrm{M}+\mathrm{H}]^{+}$; and the adduct ions selected for data collection in the $\mathrm{ESI}^{-}$mode included $[\mathrm{M}-\mathrm{H}]^{-},[\mathrm{M}+\mathrm{Na}-2 \mathrm{H}]^{-},[\mathrm{M}-2 \mathrm{H}]^{2-}$, $[\mathrm{M}+\mathrm{HCOO}]^{-}$, and $[\mathrm{M}+\mathrm{Cl}]^{-}$. Peak alignment was carried out using all runs in the experiment as reference. Peak picking threshold of sensitivity was set at 3 . All data were normalized to the summed total ion intensity per chromatogram.

The obtained data matrices were imported into MetaboAnalyst 4.0 (https://www.metaboanalyst.ca), a web server designed to facilitate analysis of metabolites and data pre-processing, including missing value estimation, median centering, log-transformation, and $\mathrm{Pa}$ reto scaling. Features existed in at least $50 \%$ of samples, and features with coefficient of variation less than $25 \%$ in the QC samples were further analyzed (Liu et al., 2020). Nonparametric tests (Wilcoxon rank-sum tests) 
Table 1. Acidity and viable counts of milk and brown milk fermented by Lactobacillus bulgaricus ND02 ( \pm $\mathrm{SD})$

\begin{tabular}{lcccc}
\hline Sample & $\begin{array}{c}\text { Fermentation } \\
\text { time }(\mathrm{h})\end{array}$ & $\mathrm{pH}$ & $\begin{array}{c}\text { Titratable acidity } \\
\left({ }^{\circ} \mathrm{T}\right)\end{array}$ & $\begin{array}{c}\text { Viable counts } \\
\left(\times 10^{8} \mathrm{cfu} / \mathrm{mL}\right)\end{array}$ \\
\hline Fermented milk & $7.00 \pm 0.58$ & $4.49 \pm 0.02$ & $86.24 \pm 0.80$ & $5.15 \pm 0.04$ \\
Fermented brown milk & $7.20 \pm 0.20$ & $4.50 \pm 0.00$ & $83.12 \pm 0.41$ & $8.54 \pm 0.10$ \\
\hline
\end{tabular}

were used to evaluate the significance of variables. The adjusted $P$-value (false discovery rate) cutoff was set as 0.05 before further pattern recognition analysis (principal component analysis, PCA; orthogonal partial least square discriminant analysis, OPLS-DA; Liu et al., 2020). To visualize group-based clustering of metabolites, OPLS-DA was used. Differential metabolites were defined as follows: variable important in projection (VIP) value $>1$ in OPLS-DA analysis; $P<0.05$ in single-dimensional statistical analysis; and fold-change between 2 groups $>2$. Furthermore, a permutation test was also performed to assess the quality of the OPLSDA models. The goodness-of-fit was represented by $\mathrm{R}^{2} \mathrm{Y}$, and the predictive ability was indicated by $\mathrm{Q}^{2}$.

Metabolite Annotation and Pathway Analysis. Pathway enrichment analysis and metabolite annotation were performed using Mummichog 5 (Li et al., 2013a). The ".csv" files were input as 3 columns, corresponding to $m / z$ features, $P$-values, and statistical scores of 2 sample groups in both $\mathrm{ESI}^{+}$and $\mathrm{ESI}^{-}$modes, into Mummichog to conduct pathway and module analysis. The Kyoto Encyclopedia of Genes and Genomes Bacillus subtilis pathway library was selected, and the mass accuracy of instrument was $5 \mathrm{ppm}$. The $P$-value cutoff was set to 0.05 to identify significantly enriched $m / z$ features. Default settings were applied to other parameters. The results of the metabolite annotation and Mummichog pathway analysis were presented by network visualization analysis. Identified differential metabolites were subjected to MetaboAnalyst 4.0 for exploratory receiver operating characteristic (ROC) analysis. Random forest algorithms were used for ROC curve construction (Liu et al., 2020).

\section{RESULTS}

\section{Acidity and Viable Counts of Fermented Milk and Fermented Brown Milk}

The acidity and viable counts of milk and brown milk fermentation by L. bulgaricus ND02 are shown in Table 1. The $\mathrm{pH}$ decreased within the process of fermentation of milk and brown milk by L. bulgaricus ND02, and the TA gradually increased; the acidification continued until reaching the fermentation endpoint of $\mathrm{pH}$
4.5 after around $7 \mathrm{~h}$. The TA $\left({ }^{\circ} \mathrm{T}\right)$ of fermented milk and fermented brown milk was $86.24^{\circ} \mathrm{T}$ and $83.12^{\circ} \mathrm{T}$, respectively; both were between 70 and $110^{\circ} \mathrm{T}$, indicating that L. bulgaricus ND02 had the ability to produce acid and the acidity was within an acceptable range for consumers (Sudun et al., 2013). The viable count of the LAB directly affected the quality and function of fermented dairy products, and the viable counts of L. bulgaricus ND02 in both the fermented milk and brown fermented milk were above $10^{8} \mathrm{cfu} / \mathrm{mL}$, indicating that L. bulgaricus ND02 had good fermentation performance.

\section{Analysis of UPLC-Q-TOF MS ${ }^{E}$ Chromatograms}

Typical base peak intensity chromatograms of the 4 types of milk samples, including milk, brown milk, fermented milk, and fermented brown milk, are shown in Figure 1. The chromatograms depict the changes in intensity of detected ion signals during each run, and they reflect the overall information of the sample. The chromatographic profiles of the fermented milk and fermented brown milk were highly similar, and those of milk and brown milk were also similar to each other. However, significant differences were seen in the chromatographic profiles between nonfermented and fermented milks.

\section{Metabolomics Analysis}

Quality Control. A QC standard was prepared by pooling equal aliquots of all samples. The injections showed a stable run condition with small variation (less than $\pm 2 \mathrm{SD}$; Figure 2A). The close clustering of the QC samples on the PCA score plot confirmed that the chromatographic runs were stable and reproducible (Figure 2B; Figure 3). The 4 sample groups clustered distinctly on the PCA score plot on $\mathrm{ESI}^{+}$, and most sample groups except fermented milk clustered closely on the PCA score plot on ESI- (Figure 3).

Changes in the Milk Metabolome After the Maillard Reaction. A total of 2,523 spectral features were yielded in the milk and brown milk metabolomes generated in $\mathrm{ESI}^{+}$and $\mathrm{ESI}^{-}$modes after data filtering and missing value removal. The OPLS-DA of the milk 
and brown milk metabolomes was performed (Figure $4 \mathrm{~A}$ ), which was a supervised modeling method. The milk samples were located distantly from the brown milk samples, indicating that marked differences existed between the metabolomes of the 2 sample groups. In $\mathrm{ESI}^{+}$mode, the cumulative $\mathrm{R}^{2} \mathrm{X}, \mathrm{R}^{2} \mathrm{Y}$, and $\mathrm{Q}^{2}$ of the OPLS-DA between the milk and brown milk groups were $0.710,1$, and 0.958 , respectively (Table 2). In $\mathrm{ESI}^{-}$mode, the cumulative $\mathrm{R}^{2} \mathrm{X}, \mathrm{R}^{2} \mathrm{Y}$, and $\mathrm{Q}^{2}$ of the OPLS-DA between the milk and brown milk groups were $0.790,0.995$, and 0.754 , respectively (Table 2). Both OPLS-DA models showed goodness-of-fit and high prediction ability. Permutation tests were carried out to confirm the stability and robustness of the OPLS-DA models presented in this study (Figure 4B). The permutations for 200 times was applied to assess the risk that the current OPLS-DA model was spurious, and the results indicated no overfitting (Worley and Powers, 2016). To identify significant metabolites that contributed to the discrimination, an S-plot (Figure 4C) was generated from the OPLS-DA regression. In the S-plot (Figure 4C), red squares indicated substances with VIP value $>1$. The $\mathrm{S}$-plot was used to identify differential compounds based on covariance and loading profiling correlation of each metabolite. Each dot in the S-plot represented a single metabolite. Metabolites with large contribution and significant differences would be located away from the origin on the S-plot, which would be considered as potential biomarker candidates.

Significant biomarker candidates fulfilled the following criteria: VIP value $>1, P<0.05$, and fold-change $>2$. The significant differential retention time exact mass pairs obtained from Mummichog and OPLSDA predictions were selected and imported back into

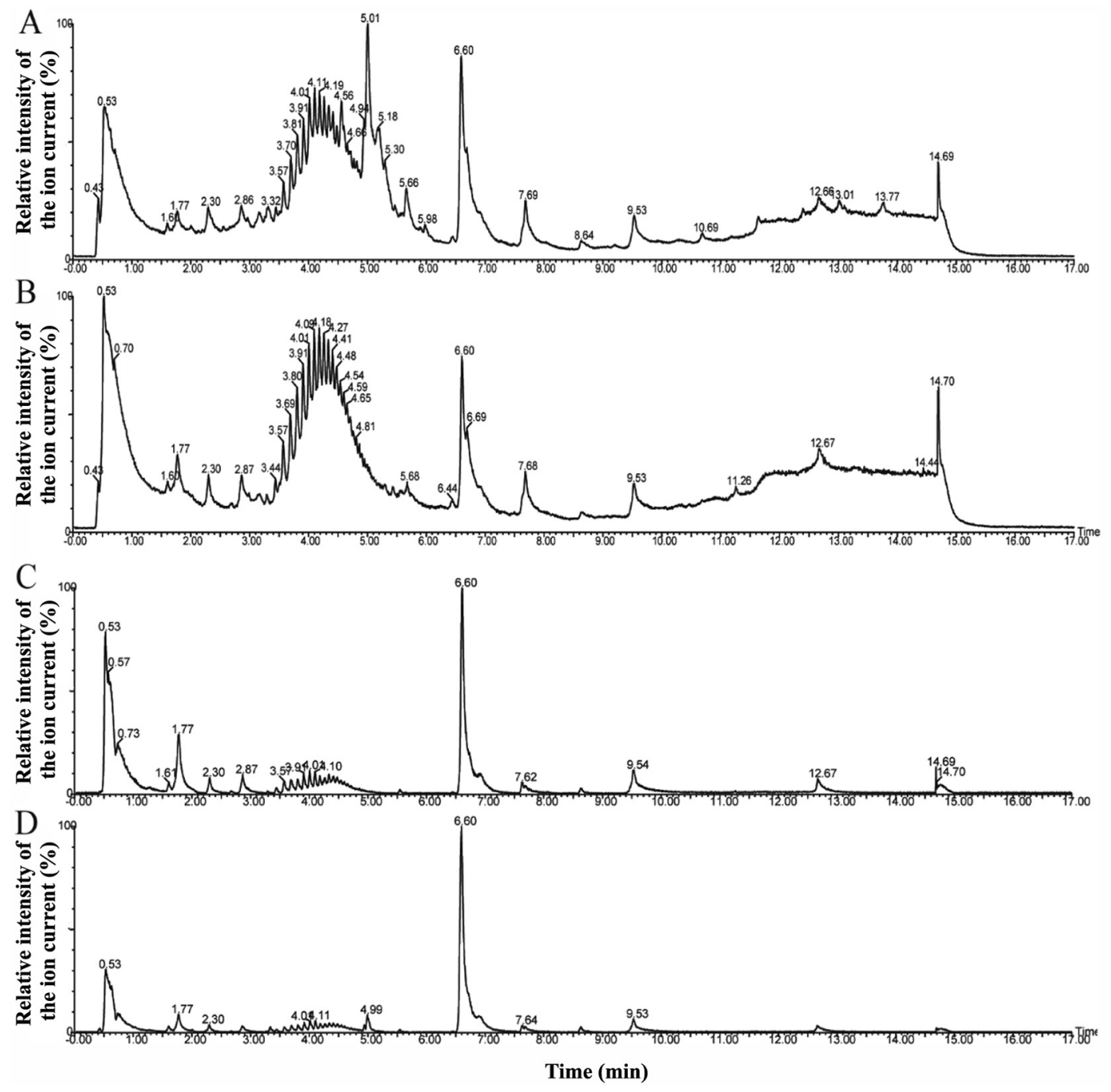

Figure 1. Representative based peak intensity chromatograms of different milk samples. (A) Milk; (B) brown milk; (C) fermented milk; (D) fermented brown milk. 

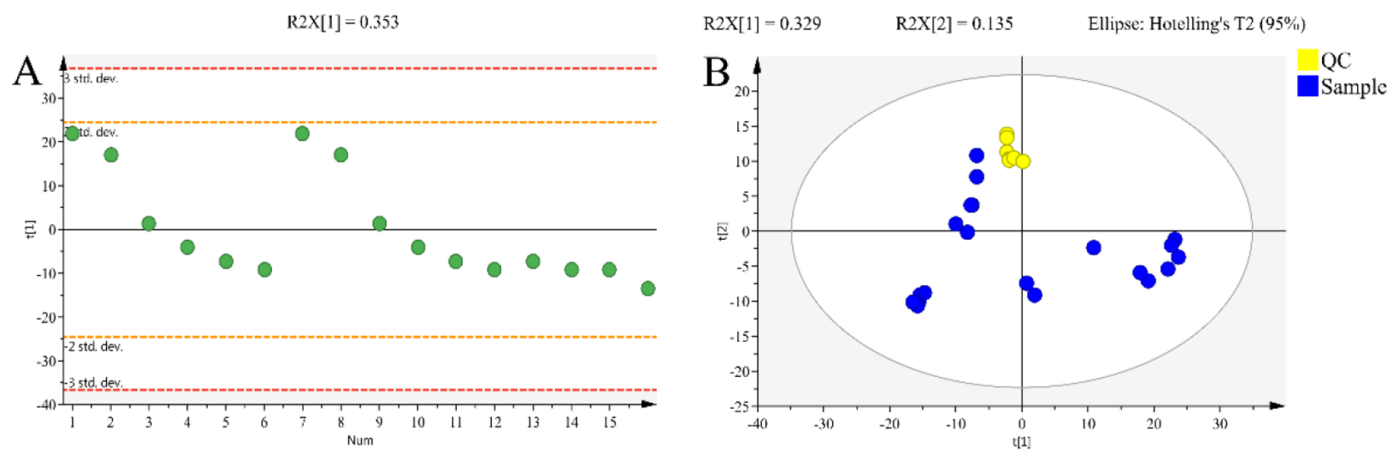

Figure 2. Assessment of quality control (QC) samples. (A) Trend plot showing the variation of t[1] against all QC runs. The x-axis represents the QC sample number, and the y-axis, $\mathrm{t}[1]$, represents the fluctuation of signal of QC samples in arbitrary units. The upper and lower ranges up to 3 SD are shown. (B) Principal component analysis (PCA) score plot of the milk samples and QC samples. $t[1]$ and $t[2]$ are the 2 most important new variables in summarizing the dataset.

Progenesis QI software for compound identification. Then, 3 individual searches were applied as follows: (1) peak picking threshold of sensitivity was 3 ; (2) the elemental composition should fit precursor tolerance within $1 \mathrm{ppm}$ difference of the candidate compounds searched by online available databases such as Chemspider (http://www.chemspider.com/) and Kyoto Encyclopedia of Genes and Genomes (https://www .kegg.jp/); and (3) fragment $m / z$ should be selected in the high-energy function $\left(\mathrm{MS}^{\mathrm{E}}\right)$. The 3 individual searches methods resulted in 56 signals from the 2,523 identified spectral features, and they were considered remarkably different between the milk and brown milk groups. A total of 20 robust known chemical markers
(Table 3) were identified. The diagnostic accuracy of the differential metabolites identified between milk and brown milk samples was also evaluated, and 17 showed a good diagnostic value by area under the curve (AUC) $>0.8$ (Table 3). To visualize chemical changes after the Maillard reaction, a heatmap was generated to show the relative abundances of identified differential components in individual milk and brown milk samples (Figure 4E).

Multivariate Analysis of the Fermented Brown Milk and Brown Milk Metabolomes. Similar methods described above were used to generate OPLS-DA models and identify differential metabolites between fermented brown milk and brown milk (Figure 5A). A
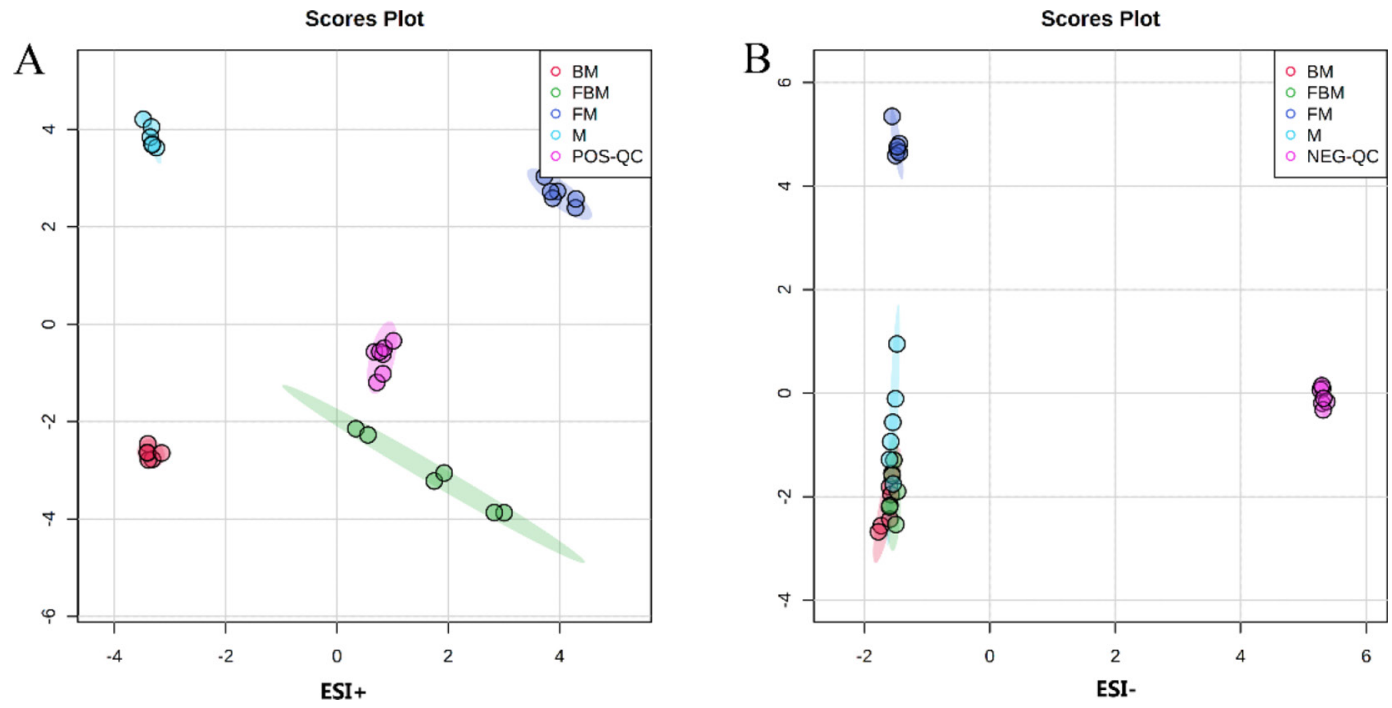

Figure 3. Principal component analysis (PCA) score plots of 4 groups of samples in (A) positive [electrospray ionization $\left.(\mathrm{ESI})^{+}\right]$and $(\mathrm{B})$ negative (ESI ${ }^{-}$) ion modes. Sample groups in this analysis: brown milk (BM), fermented brown milk (FBM), fermented milk (FM), and milk (M). QC samples run in ESI ${ }^{+}$mode and $\mathrm{ESI}^{-}$are represented by POS-QC and NEG-QC, respectively. Six samples were analyzed from each group. Each color symbol represents the metabolome of one sample. The x-axis represents the correlation coefficients of principal components and metabolites, and the y-axis represents the correlation coefficient between principal components and metabolites. 


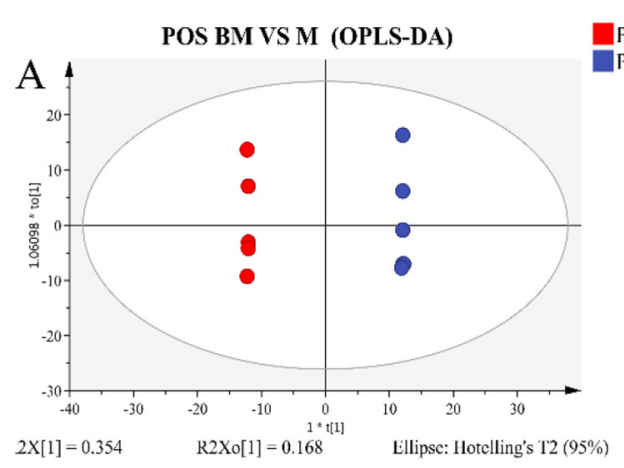

CPOS BM POS BM VS M (PLS-DA): : R2=(0.0, 0.993), Q2 $=(\mathbf{0 . 0}, \mathbf{0 . 5 4 5}) \bullet \mathrm{R} 2$
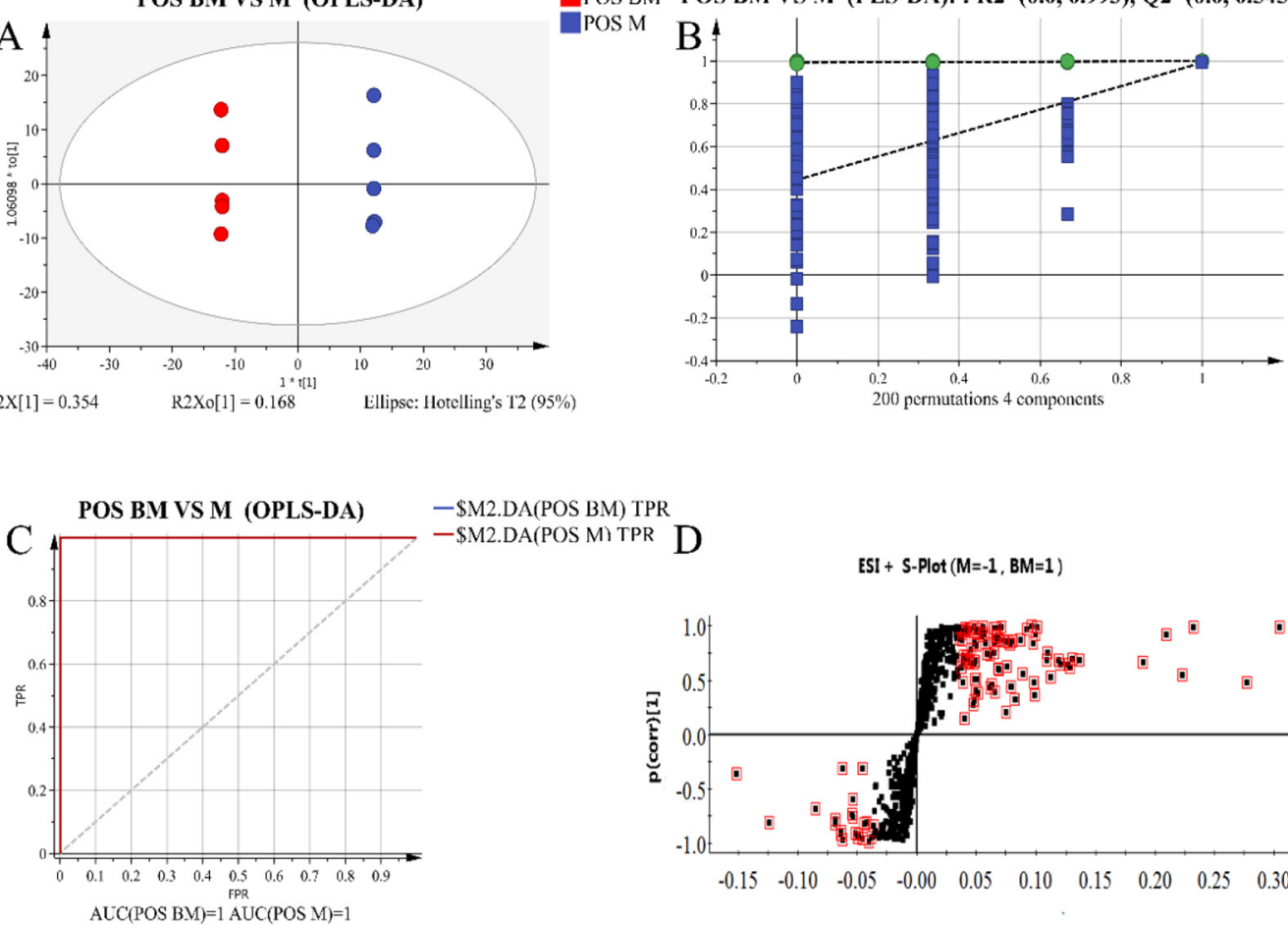

$-\$ M 2 . D A(P O S B M) T P R$ -\$M2.DA(POS M) TPR D

$\mathrm{ACC}(\mathrm{POS} B \mathrm{M})=1 \mathrm{AUCC}(\mathrm{POS} \mathrm{M})=$
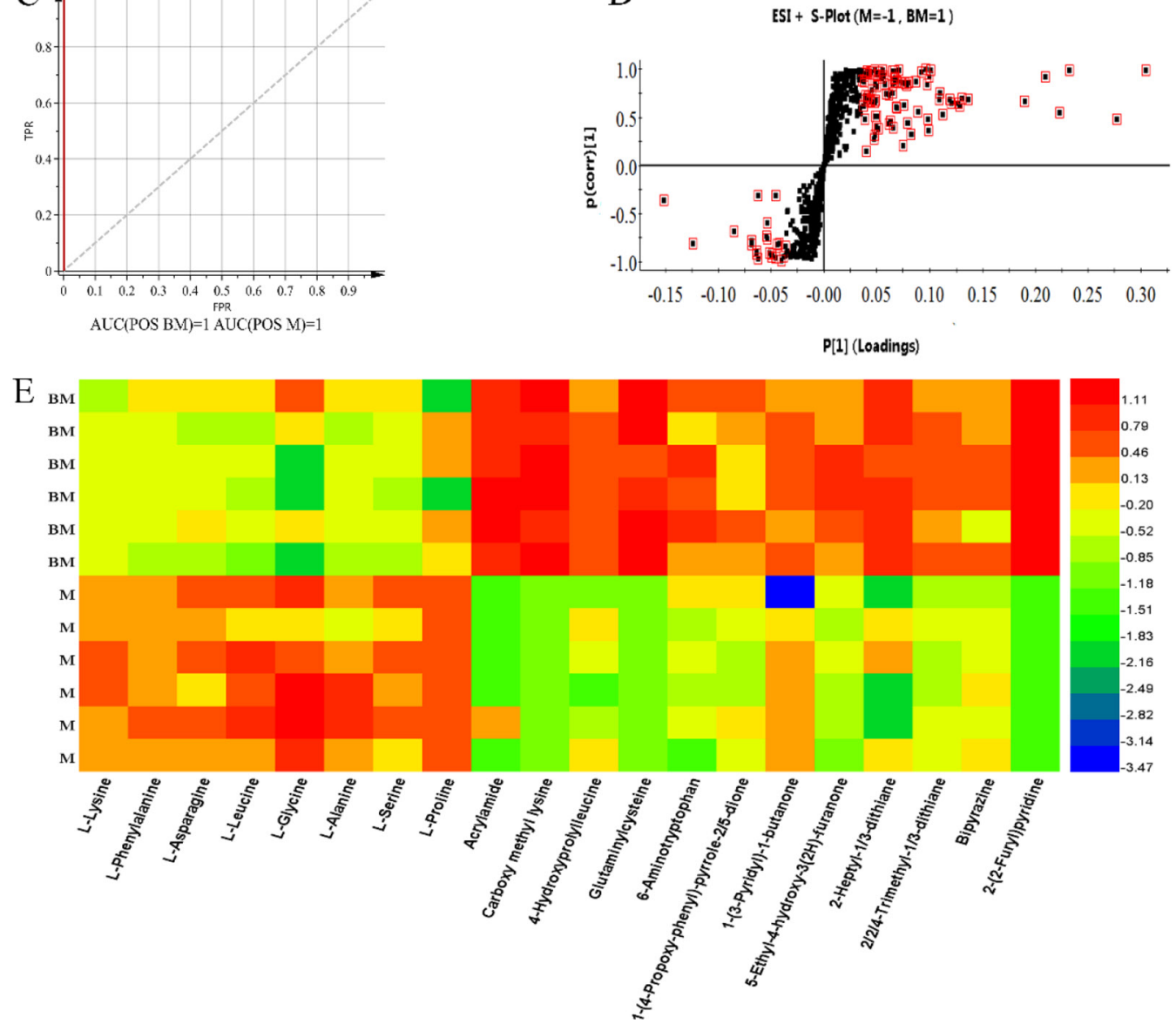

Figure 4. Metabolomic analysis of brown milk (BM) and milk (M). (A) Score plot of orthogonal partial least squares discriminant analysis (OPLS-DA), (B) permutation test (200 random permutations), (C) receiver operating characteristic (ROC) curve, and (D) S-plot of orthogonal partial least squares discriminant analysis (OPLS-DA) of BM and milk M metabolomes. Red squares indicate substances with variable importance in projection (VIP) value $>1$. (E) Heatmap showing the relative abundance of differential metabolites between BM and M. In panel B, the $\mathrm{y}$-axis represents the values of $\mathrm{R}$ and $\mathrm{Q}$, and the $\mathrm{x}$-axis is the similarity to the original model. $\mathrm{M} 2$.DA $=$ drawing number in the software. TPR $=$ true positive rate $\mathrm{POS}=$ receiver operating characteristic $(\mathrm{ROC})$ curve in positive [electrospray ionization $(\mathrm{ESI})^{+}$] ion modes. The color scale represents the relative abundance of significant differential abundant metabolites: blue indicates the lowest abundance and red the highest. The relative abundance is preprocessed by median centering, Log2 transformation, and Pareto scaling.

total of 2,809 spectral features were retained after data filtering. The OPLS-DA model achieved significant separation between groups and showed goodness-of-fit and high prediction ability $\left(\mathrm{ESI}^{+}=\mathrm{R}^{2} \mathrm{X}=0.772, \mathrm{R}^{2} \mathrm{Y}\right.$ $=1, \mathrm{Q}^{2}=0.987 ; \mathrm{ESI}^{-}: \mathrm{R}^{2} \mathrm{X}=0.726, \mathrm{R}^{2} \mathrm{Y}=0.956, \mathrm{Q}^{2}$ $=0.892 ;$ Table 2$)$. The permutation test showed that 
Table 2. Pairwise comparison of data sets generated in positive ion mode and negative ion mode using principal component analysis (PCA) and orthogonal partial least square discriminant analysis (OPLS-DA) models

\begin{tabular}{|c|c|c|c|c|}
\hline Mode and sample pair & $\begin{array}{l}\text { Statistical } \\
\text { model }\end{array}$ & $\begin{array}{c}\mathrm{R}^{2} \mathrm{X} \\
\text { (cumulative) }\end{array}$ & $\begin{array}{c}\mathrm{R}^{2} \mathrm{Y} \\
\text { (cumulative) }\end{array}$ & $\begin{array}{c}\mathrm{Q}^{2} \\
\text { (cumulative) }\end{array}$ \\
\hline \multicolumn{5}{|l|}{ Positive ion mode } \\
\hline Brown milk and milk & OPLS-DA & 0.710 & 1.000 & 0.958 \\
\hline \multirow{2}{*}{ Fermented brown milk and brown milk } & $\mathrm{PCA}$ & 0.645 & - & 0.393 \\
\hline & OPLS-DA & 0.772 & 1.000 & 0.987 \\
\hline Fermented milk and milk & PCA & 0.966 & — & 0.943 \\
\hline Fermented brown milk and fermented milk & OPLS-DA & 0.753 & 1.000 & 0.965 \\
\hline \multicolumn{5}{|l|}{ Negative ion mode } \\
\hline \multirow[t]{2}{*}{ Brown milk and milk } & PCA & 0.621 & - & 0.091 \\
\hline & OPLS-DA & 0.790 & 0.995 & 0.754 \\
\hline \multirow[t]{2}{*}{ Fermented brown milk and brown milk } & $\mathrm{PCA}$ & 0.513 & - & 0.122 \\
\hline & OPLS-DA & 0.726 & 0.956 & 0.892 \\
\hline Fermented milk and milk & $\mathrm{PCA}$ & 0.873 & - & 0.739 \\
\hline
\end{tabular}

the supervised models had good stability and robustness (Figure 5B). In the S-plots, each scattered point represented a variable, and the further away from the origin, the greater the contribution of this variable to the difference between groups. In $\mathrm{ESI}^{+}$mode, 246 metabolites showed significant deviation away from the origin (VIP value $>1$ ), which were potential differential metabolites between these 2 sample groups (Figure 5D). An individual search for metabolites of VIP value $>1$ identified a total of 493 signals from the 2,809 spectral features that showed marked difference in abundance between the fermented brown milk and brown milk. A total of 21 significant differential metabolites were further selected based on the criteria: VIP value $>1, P$ $<0.05$, and fold-change $>2$ (Table 4). Sixteen of the 21 differential metabolites showed a good diagnostic value of AUC $>0.8$, including L-lysine, Glu-Glu, and 2-methylquinolin-4-ol (Table 4). A heatmap was generated to visualize differences in the relative abundances of these differential abundant metabolites (Figure 5E).

Table 3. Significant differential metabolites between brown milk and milk ${ }^{1}$

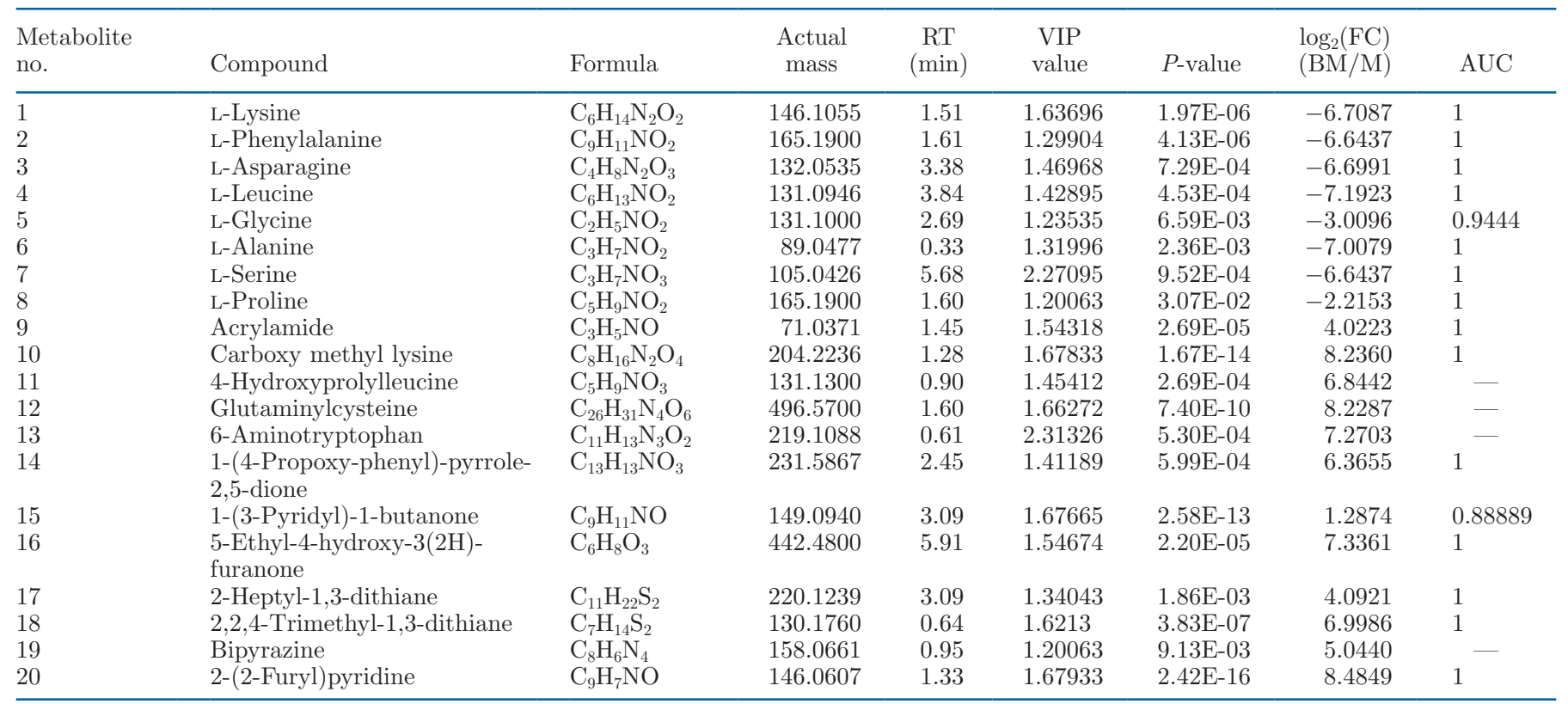

${ }^{1} \mathrm{RT}=$ retention time; $\mathrm{VIP}=$ variable importance in projection; $\mathrm{FC}=$ fold-change; $\mathrm{BM}=$ brown milk; $\mathrm{M}=$ milk; $\mathrm{AUC}=$ area under the curve (only metabolites with AUC value $>0.8$ are shown). 
Multivariate Analysis of the Fermented Milk and Milk Metabolomes. Multivariate analysis was performed between the fermented milk and milk me- tabolomes (Figure 6). In total, 2,989 spectral features were retained after data filtering. The OPLS-DA model achieved significant separation between groups. For the
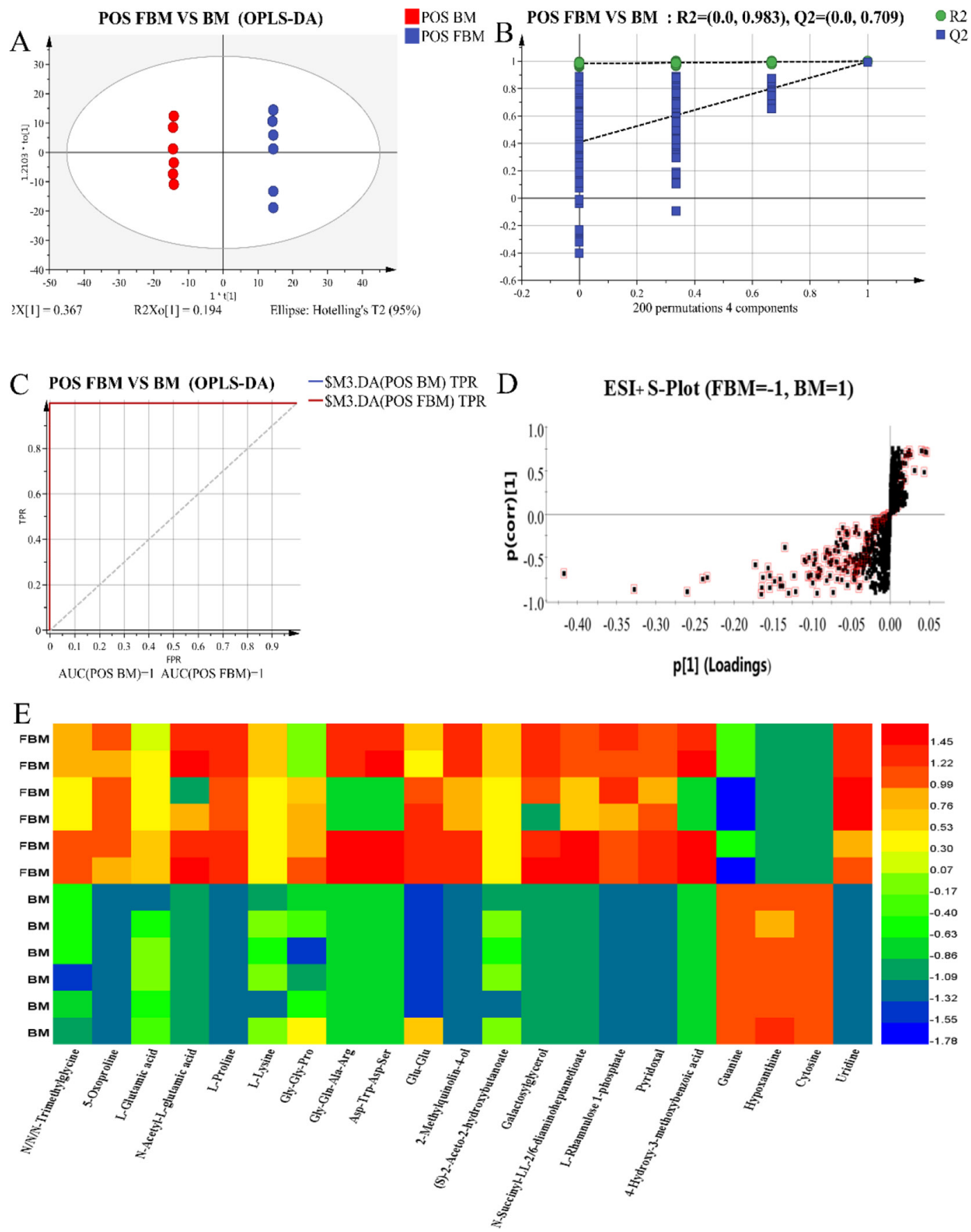

Figure 5. Metabolomic analysis of fermented brown milk (FBM) and brown milk (BM). (A) Score plot of orthogonal partial least squares discriminant analysis (OPLS-DA), (B) permutation test (200 random permutations), (C) receiver operating characteristic (ROC) curve, and (D) S-plot of orthogonal partial least squares discriminant analysis (OPLS-DA) of FBM and BM metabolomes. Red squares indicate substances with variable importance in projection (VIP) value $>1$. (E) Heatmap showing the relative abundance of differential metabolites between fermented brown milk and brown milk. In panel $\mathrm{B}$, the $\mathrm{y}$-axis represents the values of $\mathrm{R}$ and $\mathrm{Q}$, and the $\mathrm{x}$-axis is the similarity to the original model. TPR $=$ true positive rate. $\mathrm{POS}=$ receiver operating characteristic $(\mathrm{ROC})$ curve in positive [electrospray ionization $(\mathrm{ESI})^{+}$] ion modes. The color scale represents the relative abundance of significant differential abundant metabolites: blue indicates the lowest abundance and red the highest. The relative abundance is preprocessed by median centering, Log2 transformation, and Pareto scaling. 
Peng et al.: FERMENTED BROWN MILK METABOLOMES

Table 4. Significant differential metabolites between fermented brown milk and brown milk ${ }^{1}$

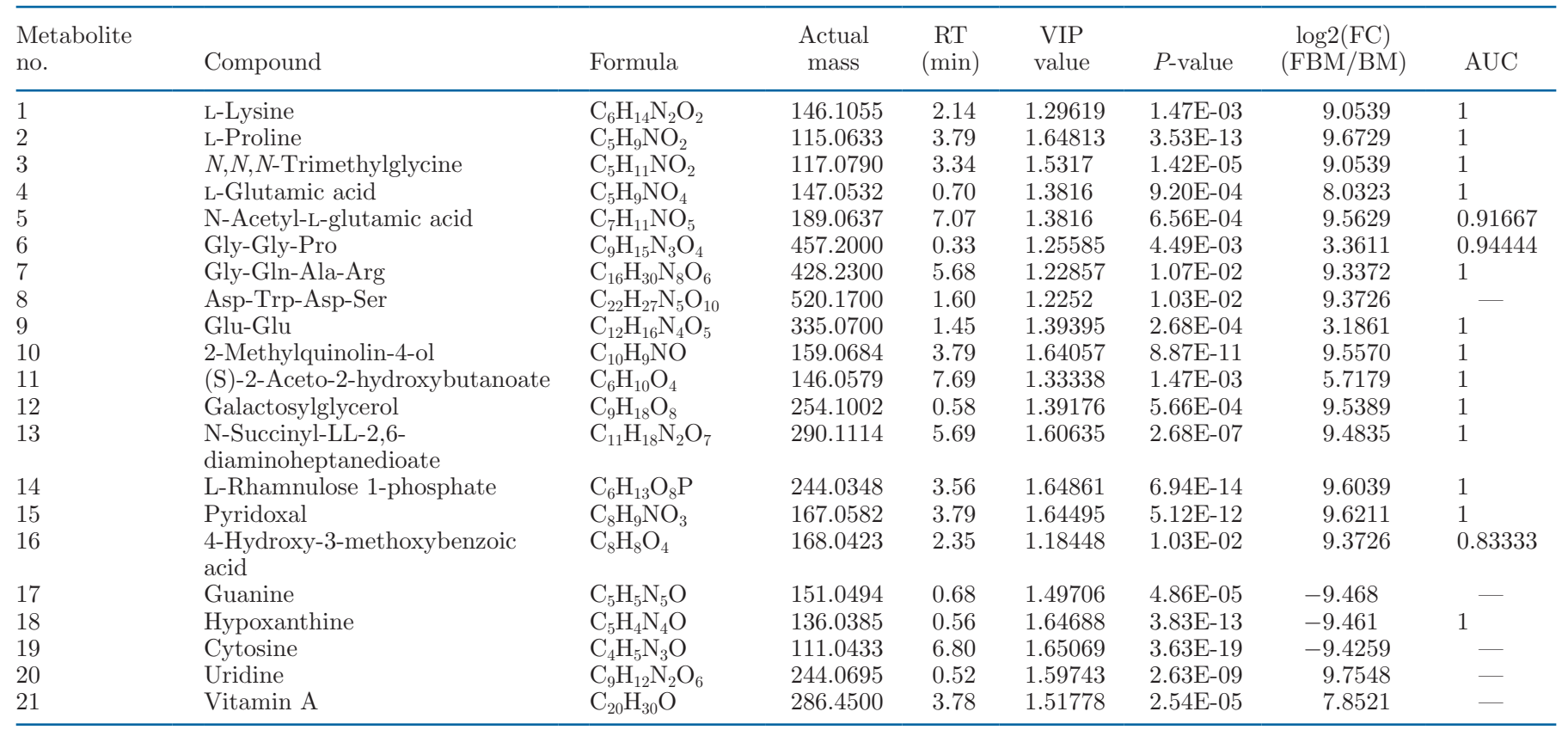

${ }^{1} \mathrm{RT}=$ retention time; $\mathrm{VIP}=$ variable important in the projection; $\mathrm{FC}=$ fold-change; $\mathrm{FBM}=$ fermented brown milk; $\mathrm{BM}=$ brown milk; $\mathrm{AUC}$ $=$ area under the curve (only metabolites with AUC value $>0.8$ are shown).

$\mathrm{ESI}^{+}$mode, the cumulative $\mathrm{R}^{2} \mathrm{X}, \mathrm{R}^{2} \mathrm{Y}$, and $\mathrm{Q}^{2}$ of the OPLS-DA model between the fermented milk and milk group were $0.990,0.995$, and 0.995 , respectively (Table 2 ). For the $\mathrm{ESI}^{-}$, the cumulative $\mathrm{R}^{2} \mathrm{X}, \mathrm{R}^{2} \mathrm{Y}$, and $\mathrm{Q}^{2}$ of the OPLS-DA model between these 2 sample groups were $0.965,0.960$, and 0.820 , respectively (Table 2 ). Both OPLS-DA models showed goodness-of-fit and high prediction ability (Figure 6B). The S-plot showed a plurality of ions away from the origin, indicating the presence of differential metabolites between the fermented milk and milk samples (Figure 6D).

The 3 individual searches found 68 signals out of the 2,809 spectral features that showed marked differential abundance between these 2 milk groups. A total of 23 robust chemical markers were identified (Table 5). Seventeen of these differential metabolites showed good diagnostic value with AUC $>0.8$ (Table 5). A heatmap was generated to visualize differences in the relative abundances of these differential abundant metabolites (Figure 6E).

Multivariate Analysis of Fermented Brown Milk and Fermented Milk Metabolomes. Multivariate analysis was performed between the fermented brown milk and fermented milk metabolomes (Figure 7). A total of 1,621 spectral features were retained after data filtering. The OPLS-DA models of the fermented milk and the milk groups achieved significant separation in both $\mathrm{ESI}^{+}$mode (cumulative $\mathrm{R}^{2} \mathrm{X}, \mathrm{R}^{2} \mathrm{Y}$, and $\mathrm{Q}^{2}$ were $0.753,1$, and 0.965 , respectively; Table 2 ) and
$\mathrm{ESI}^{-}$mode (cumulative $\mathrm{R}^{2} \mathrm{X}, \mathrm{R}^{2} \mathrm{Y}$, and $\mathrm{Q}^{2}$ were 0.776 , 1 , and 0.964, respectively; Table 2). Both OPLS-DA models showed goodness-of-fit and high prediction ability. The permutation test showed that the supervised models had good stability and robustness (Figure 7B). The S-plot found a plurality of ions away from the origin, suggesting the presence of differential metabolites between these 2 sample groups (Figure 7D). By performing the 3 metabolite searches described above, a total of 16 significant differential metabolites were identified between these 2 sample groups (Table 6), and 6 of these showed a high AUC value $>0.8$ (Table 6). A heatmap was generated to visualize differences in the relative abundances of these differential abundant metabolites (Figure 7E). Differences in the abundance of these 6 metabolites between the 2 fermented milk types are shown in Figure 8A, and the ROC curves of these differential metabolites are shown in Figure 8B.

Prediction of Mummichog Pathway Activity Between Sample Pairs. The Mummichog pathway activity was predicted between different sample pairs (Figure 9). Pathways related to pyrimidine metabolism, lysine biosynthesis, and pantothenate and CoA biosynthesis were enriched in the metabolomes of the fermented brown milk compared with brown milk ( $P$ $<0.05$; Figure 9A). Pathways related to valine, leucine and isoleucine degradation, riboflavin metabolism, and phenylalanine metabolism were enriched in the metabolomes of the fermented milk compared with milk 

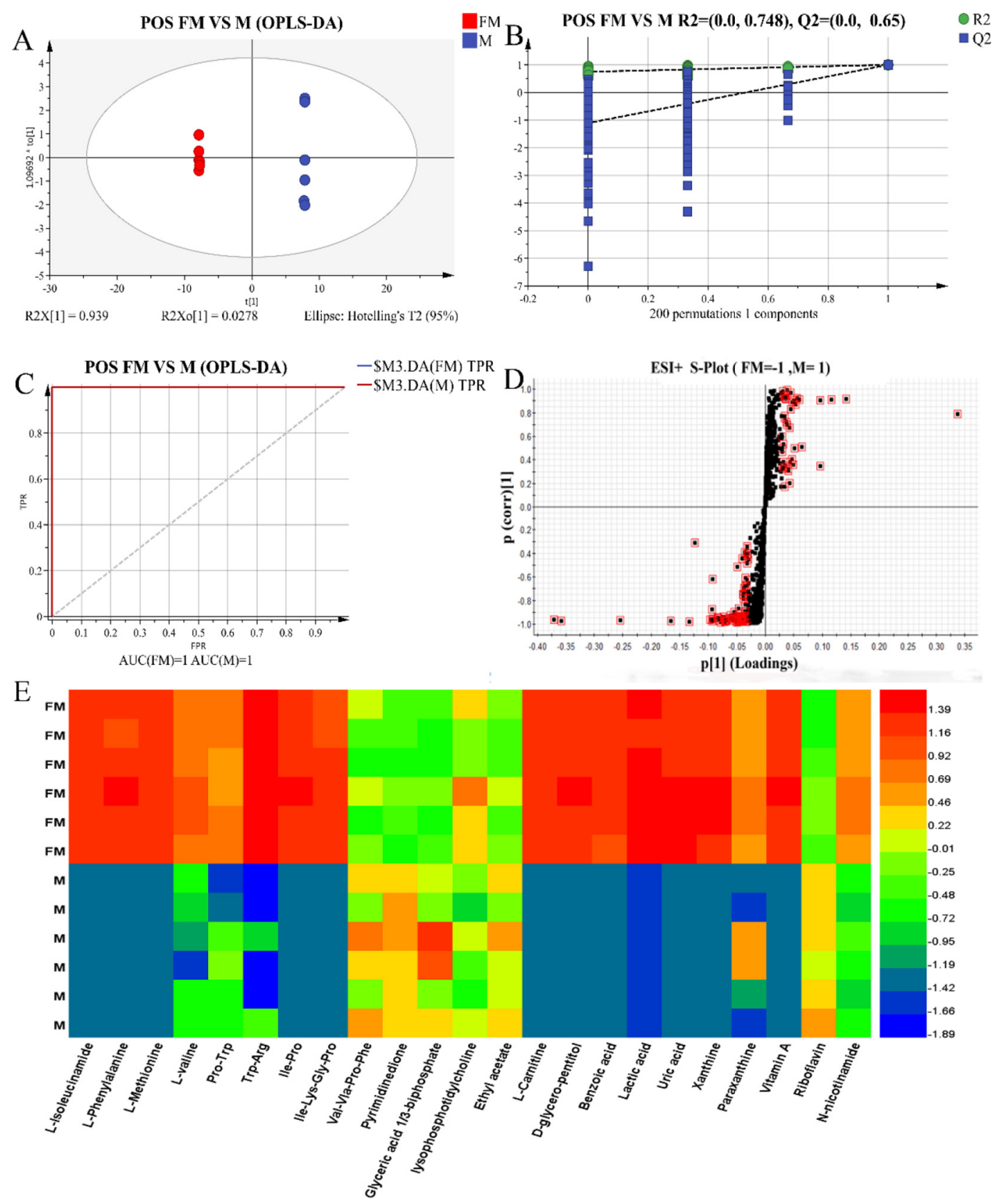

Figure 6. Metabolomic analysis of fermented milk (FM) and milk (M). (A) Score plot of orthogonal partial least squares discriminant analysis (OPLS-DA), (B) permutation test (200 random permutations), (C) receiver operating characteristic (ROC) curve, and (D) S-plot of orthogonal partial least squares discriminant analysis (OPLS-DA) of FM and M metabolomes. Red squares indicate substances with variable importance in projection (VIP) value $>1$. (E) Heatmap showing the relative abundance of differential metabolites between fermented milk and milk. In panel $\mathrm{B}$, the $\mathrm{y}$-axis represents the values of $\mathrm{R}$ and $\mathrm{Q}$, and the $\mathrm{x}$-axis is the similarity to the original model. TPR $=$ true positive rate. $\mathrm{POS}=$ receiver operating characteristic $(\mathrm{ROC})$ curve in positive [electrospray ionization $(\mathrm{ESI})^{+}$] ion modes. The color scale represents the relative abundance of significant differential abundant metabolites: blue indicates the lowest abundance and red the highest. The relative abundance is preprocessed by median centering, Log2 transformation, and Pareto scaling.

$(P<0.05 ;$ Figure 9B). Pathways related to taurine and hypotaurine metabolism, pyrimidine metabolism, and lysine biosynthesis were enriched in the metabolomes of the fermented brown milk compared with fermented milk $(P<0.05$; Figure 9C).

\section{DISCUSSION}

Fermented dairy products are popular worldwide, and they have good nutritional values. In recent years, new fermented milk products have been developed 
(e.g., fermented brown milk, which has a unique flavor, rich aroma, and nutrition). To understand the chemical nature of fermented brown milk, this work explored the metabolomic differences between milk and brown milk to describe chemical changes that occurred during the Maillard reaction. Meanwhile, the metabolomic profiles between fermented milk and fermented brown milk were compared to identify differential metabolites responsible for the unique features of fermented brown milk.

\section{Metabolomic Changes During the Maillard Reaction}

The reconstituted milk was heated to $95^{\circ} \mathrm{C}$ for $3 \mathrm{~h}$ for browning to occur. Then, LC-MS was used to analyze differential metabolites between brown milk and milk samples. Significant changes in the milk metabolomes occurred after browning, and 21 significant differential metabolites were detected, which mainly belonged to the following: AA, peptides, bovine milk lipids, organic acids, nucleosides, vitamins, aldoketones, dithianes, pyrazines, pyrralines, and amide compounds.

Significant changes occurred in the milk AA composition after the Maillard reaction took place. Eight free AA, including L-lysine, L-phenylalanine, L-asparagine, L-leucine, L-glycine, L-alanine, L-serine, and L-proline, significantly decreased in the brown milk compared with milk (Table 3; Figure 4E). Milk is a highly perishable food material that easily undergoes physical, chemical, and nutritional changes upon processing. Heat treatment of milk leads to protein degradation, and chemical changes occur between the reducing sugars (mainly lactose, glucose, and galactose) and amino groups of the protein (mainly lysine in milk $\mathrm{CN}$ and whey proteins) in nonenzymatic Maillard browning (Gu et al., 2009). When both components are present, the reaction might occur. Particularly, the content of lysine significantly decreases in the Maillard browning process. The Maillard reaction is divided into the following 3 stages: primary, advanced, and final stages. During the early stage, lactose interacts with lysine and the reaction continues, forming a Schiff's base and undergoing Amadori rearrangement to constitute lactulosyllysine (Aalaei et al., 2019). The reactivity of free AA with glucose ranks as follows: lysine $>$ glycine $>$ tryptophan $>$ tyrosine $>$ histidine $>$ arginine $>$ cysteine (Ashoor and Zent, 1984).

Moreover, our data showed that there were more AA derivatives in brown milk compared with milk, including acrylamide, carboxymethyl lysine, 4-hydroxyprolylleucine, glutaminylcysteine, and 6-aminotryptophan. Acrylamide can be released by thermal treatment of certain AA (e.g., asparagine), particularly in combination with reducing sugars, and Maillard-driven

Table 5. Significant differential metabolites between fermented milk and milk ${ }^{1}$

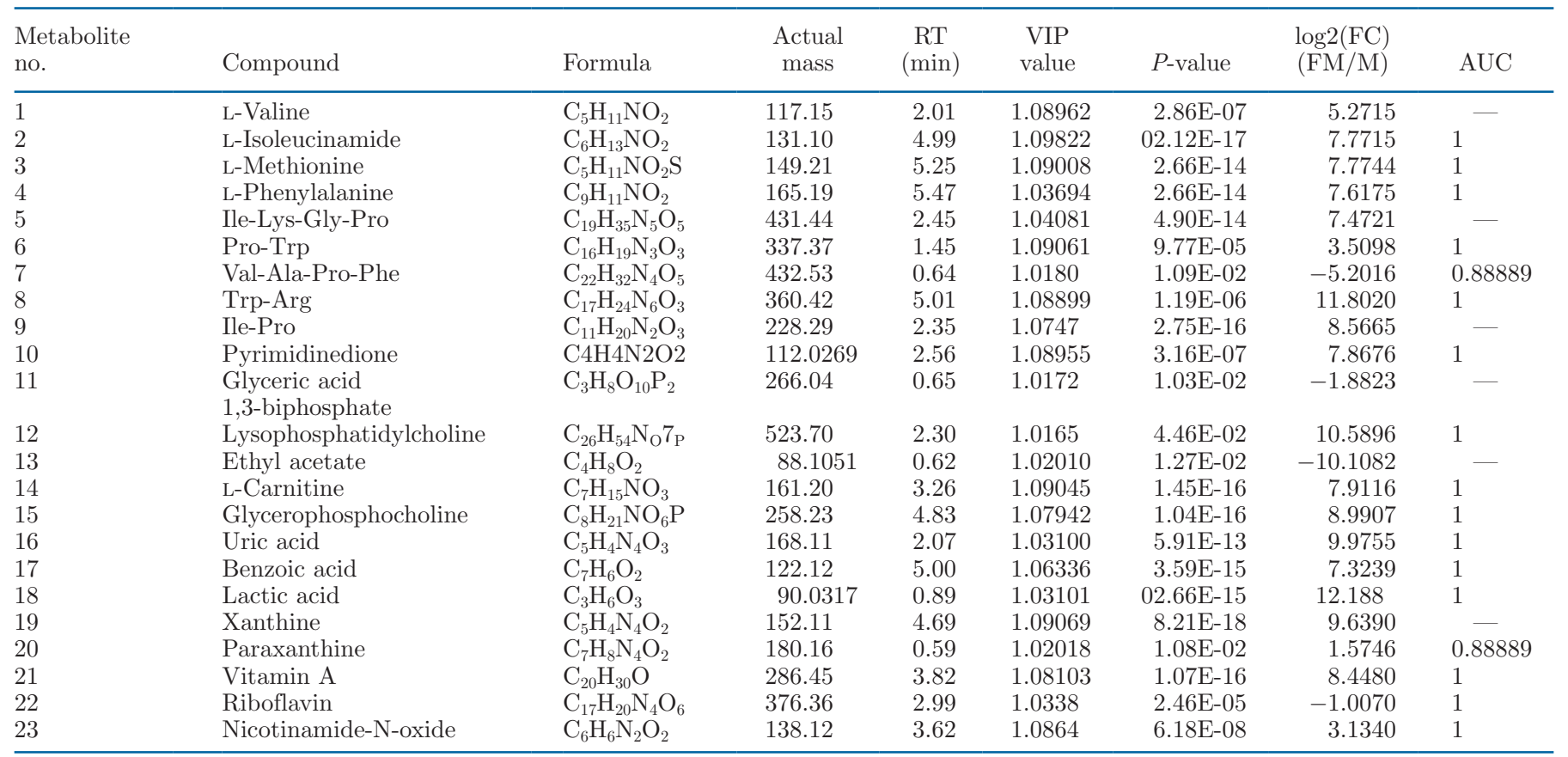

${ }^{1} \mathrm{RT}=$ retention time; $\mathrm{VIP}=$ variable important in the projection; $\mathrm{FC}=$ fold-change; $\mathrm{FM}=$ fermented milk; $\mathrm{M}=$ milk; $\mathrm{AUC}=$ area under the curve (only metabolites with AUC value $>0.8$ are shown). 
acrylamide generation influences the flavor and color in thermally processed milk (Mottram et al., 2002). Some compounds formed during the Maillard reaction have caused debates related to health concerns (e.g., carboxymethyl lysine, an Amadori degradation compound; Aalaei et al., 2019).
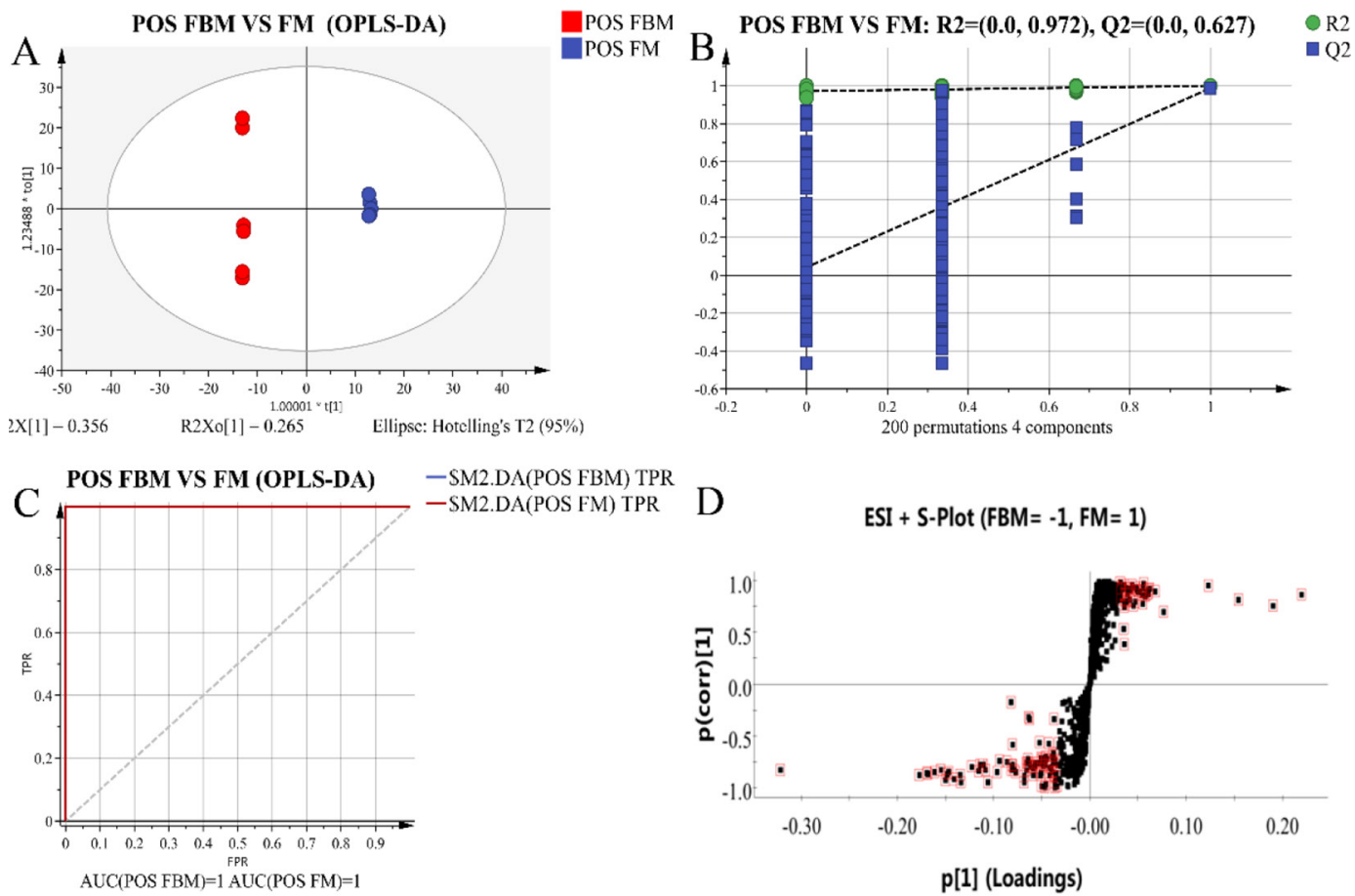

E

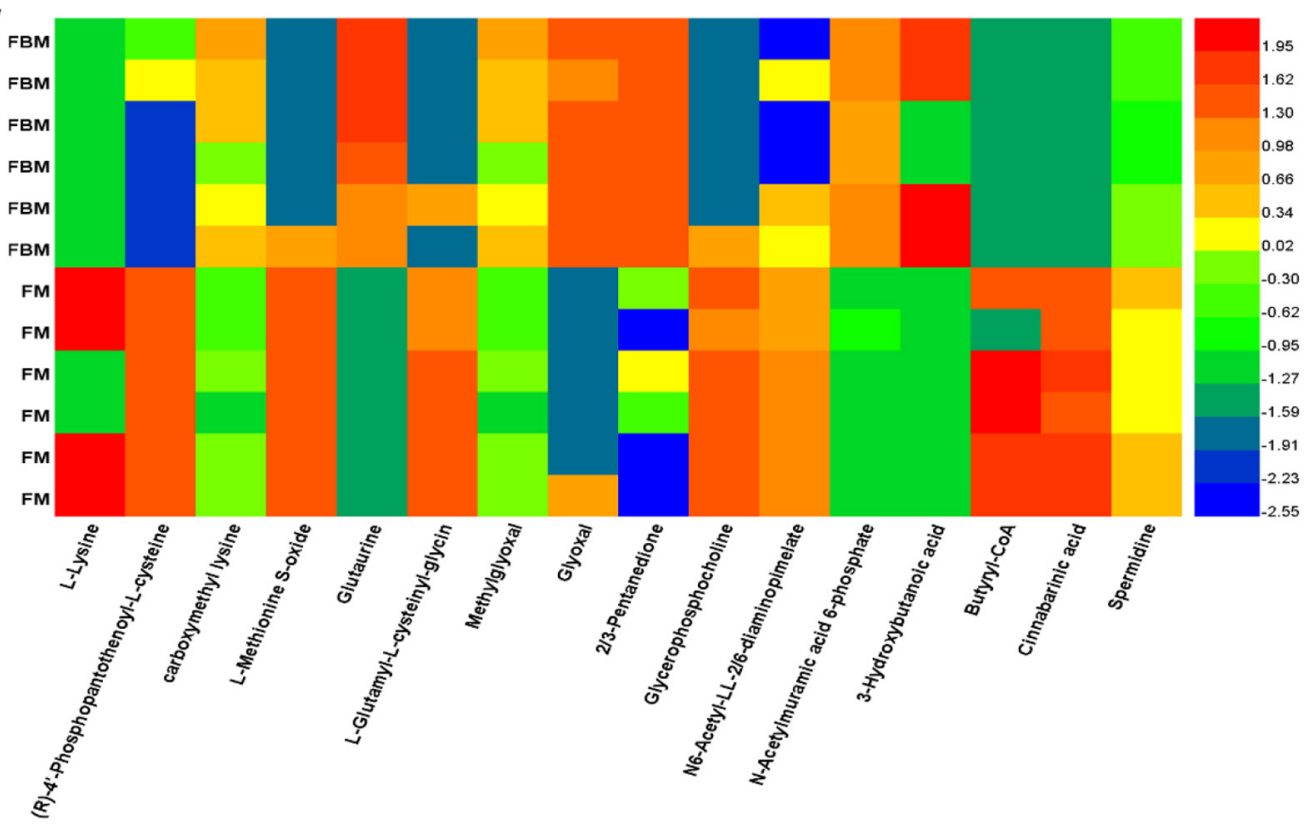

Figure 7. Metabolomic analysis of fermented brown milk (FBM) and fermented milk (FM). (A) Score plot of orthogonal partial least squares discriminant analysis (OPLS-DA), (B) permutation test (200 random permutations), (C) receiver operating characteristic (ROC) curve, and (D) S-plot of orthogonal partial least squares discriminant analysis (OPLS-DA) of FBM and FM metabolomes. Red squares indicate substances with variable importance in projection (VIP) value $>1$. (E) Heatmap showing the relative abundance of differential metabolites between fermented brown milk and fermented milk. In panel $\mathrm{B}$, the $\mathrm{y}$-axis represents the values of $\mathrm{R}$ and $\mathrm{Q}$, and the $\mathrm{x}$-axis is the similarity to the original model. $\mathrm{TPR}=$ true positive rate. $\mathrm{POS}=$ receiver operating characteristic $(\mathrm{ROC})$ curve in positive [electrospray ionization $(\mathrm{ESI})^{+}$] ion modes. The color scale represents the relative abundance of significant differential abundant metabolites: blue indicates the lowest abundance and red the highest. The relative abundance is preprocessed by median centering, Log2 transformation, and Pareto scaling. 
Our results showed that there were generally more flavor components in the brown milk than in milk, including aldehydes, ketones, alcohol, esters, organic acids, and furan compounds. The 2,3 enolization route or 1-deoxyosone-pathway occurs under neutral conditions in the advanced stage of the Maillard reaction and produces 1-deoxyketo aldose, which further produces reducing ketones and dehydro-reducing ketones, such as $\beta$-pyranone, 3 -furanone, reductones, $\alpha$-dicarbonyls, cyclopentenone, galactosylisomaltol, and acetylpyrrole (Aalaei et al., 2019). These are common chemical reactions that happen in dairy products of near-neutral $\mathrm{pH}$ in the presence of lactose. Flavor components, such as acetaldehyde, 2-furaldehyde, and furfural, are produced during the browning stage, and they provide the characteristic flavor of these products (O'Brien, 2009). Our study did not detect the entire spectrum of flavor substances mentioned above. However, significantly more aldehydes and ketones were found in the brown milk, including 1-(4-propoxy-phenyl)-pyrrole-2,5-dione, 1-(3-pyridyl)-1-butanone, and 5-ethyl-4-hydroxy-3(2H)furanone. The contribution of the detected compounds of the brown milk remains to be explored.

Apart from aldehydes, ketones, lactones, and nitrogen-containing compounds, the flavor compounds in dairy products might also include sulfur-containing compounds. Higher levels of sulfur compounds (2-heptyl-1,3-dithiane and 2,2,4-trimethyl-1,3-dithiane) were detected in brown milk compared with milk. The carbonyl intermediates of the Maillard reaction might react with sulfur compounds released in heated milk (Yu et al., 2012); however, the interactions between sulfhydryl compounds formed in milk and the intermediates derived from the Maillard reaction are not well-characterized. The strong odor of low-molecularweight sulfur compounds might also impart special organoleptic properties to brown milk (O'Brien, 2009). Other typical sulfur-containing flavor compounds in milk and dairy products are methyl mercaptan, dimethyl disulfide, and dimethyl sulfide. Although the 2 sulfur-containing compounds detected in this study (i.e., 2-heptyl-1,3-dithiane and 2,2,4-trimethyl-1,3-dithiane) have not been reported in literature, they likely contribute to the unique flavor of brown milk.

\section{Distinction Between Fermented Brown Milk and Fermented Milk Metabolomes}

Different from traditional drinking yogurt, the production of brown dairy products requires a hightemperature Maillard reaction that alters the milk components before fermentation. Thus, the starter bacteria interact with milk and brown milk differently to impart the unique color and aroma to fermented brown milk (Ni et al., 2017). It was anticipated that the 2 types of fermented milk products would have rather different metabolomic profiles. Our LC-MS analysis identified 8 types of significant differential metabolites or potential discriminatory biomarkers between the 2 types of fermented milk, involving pathways related to

Table 6. Significant differential metabolites between fermented brown milk and fermented milk ${ }^{1}$

\begin{tabular}{|c|c|c|c|c|c|c|c|c|}
\hline $\begin{array}{l}\text { Metabolite } \\
\text { no. }\end{array}$ & Compound & Formula & $\begin{array}{l}\text { Actual } \\
\text { mass }\end{array}$ & $\begin{array}{l}\mathrm{RT} \\
(\min )\end{array}$ & $\begin{array}{l}\text { VIP } \\
\text { value }\end{array}$ & $P$-value & $\begin{array}{c}\log 2(\mathrm{FC}) \\
(\mathrm{FBM} / \mathrm{FM})\end{array}$ & AUC \\
\hline 1 & L-Lysine & $\mathrm{C}_{6} \mathrm{H}_{14} \mathrm{~N}_{2} \mathrm{O}_{2}$ & 146.1055 & 5.59 & 1.18265 & $1.02 \mathrm{E}-02$ & -8.2062 & 0.88883 \\
\hline 2 & $\begin{array}{l}(\mathrm{R})-4^{\prime}- \\
\text { Phosphopantothenoyl-L- } \\
\text { cysteine }\end{array}$ & $\mathrm{C}_{12} \mathrm{H}_{23} \mathrm{~N}_{2} \mathrm{O}_{9} \mathrm{PS}$ & 402.0862 & 2.14 & 1.50885 & $7.18 \mathrm{E}-05$ & -4.8768 & - \\
\hline 3 & Carboxymethyl lysine & $\mathrm{C}_{8} \mathrm{H}_{16} \mathrm{~N}_{2} \mathrm{O}_{4}$ & 204.2236 & 3.21 & 1.35892 & $1.38 \mathrm{E}-03$ & 6.4056 & - \\
\hline 4 & L-Methionine S-oxide & $\mathrm{C}_{5} \mathrm{H}_{11} \mathrm{NO}_{3} \mathrm{~S}$ & 165.046 & 5.25 & 1.51815 & $5.04 \mathrm{E}-05$ & -3.6014 & - \\
\hline 5 & Glutaurine & $\mathrm{C}_{7} \mathrm{H}_{14} \mathrm{~N}_{2} \mathrm{O}_{6} \mathrm{~S}$ & 254.0573 & 10.46 & 1.66636 & $5.96 \mathrm{E}-11$ & 12.8077 & 1 \\
\hline 6 & $\begin{array}{l}\text { L-Glutamyl-L-cysteinyl- } \\
\text { glycin }\end{array}$ & $\mathrm{C}_{10} \mathrm{H}_{17} \mathrm{~N}_{3} \mathrm{O}_{6} \mathrm{~S}$ & 307.0838 & 1.87 & 1.48246 & $1.24 \mathrm{E}-04$ & -3.18 & \\
\hline 7 & Methylglyoxal & $\mathrm{C}_{3} \mathrm{H}_{4} \mathrm{O}_{2}$ & 72.0211 & 1.56 & 1.35892 & $1.38 \mathrm{E}-03$ & 6.4056 & 1 \\
\hline 8 & Glyoxal & $\mathrm{C}_{2} \mathrm{H}_{2} \mathrm{O}_{3}$ & 74.0004 & 1.79 & 1.48157 & $1.35 \mathrm{E}-04$ & 3.1393 & 1 \\
\hline 9 & 2,3-Pentanedione & $\mathrm{C}_{5} \mathrm{H}_{8} \mathrm{O}_{2}$ & 100.1158 & 2.12 & 1.39181 & $7.88 \mathrm{E}-04$ & 4.787 & 1 \\
\hline 10 & Glycerophosphocholine & $\mathrm{C}_{8} \mathrm{H}_{21} \mathrm{NO}_{6} \mathrm{P}$ & 258.1106 & 2.50 & 1.48157 & $1.35 \mathrm{E}-04$ & -3.1393 & - \\
\hline 11 & $\begin{array}{l}\text { N6-Acetyl-LL- } 2,6- \\
\text { diaminopimelate }\end{array}$ & $\mathrm{C}_{9} \mathrm{H}_{16} \mathrm{~N}_{2} \mathrm{O}_{5}$ & 232.1059 & 1.44 & 1.19731 & $8.94 \mathrm{E}-03$ & -2.3998 & - \\
\hline 12 & $\begin{array}{l}N \text {-Acetylmuramic acid } \\
6 \text {-phosphate }\end{array}$ & $\mathrm{C}_{11} \mathrm{H}_{20} \mathrm{NO}_{11} \mathrm{P}$ & 373.0774 & 0.70 & 1.66803 & $2.73 \mathrm{E}-11$ & 7.6504 & - \\
\hline 13 & 3-Hydroxybutanoic acid & $\mathrm{C}_{4} \mathrm{H}_{8} \mathrm{O}_{3}$ & 104.0473 & 3.07 & 1.18142 & $1.02 \mathrm{E}-02$ & 8.1007 & 0.88883 \\
\hline 14 & Butyryl-CoA & $\mathrm{C}_{25} \mathrm{H}_{42} \mathrm{~N}_{7} \mathrm{O}_{17} \mathrm{P}_{3} \mathrm{~S}$ & 837.1571 & 5.21 & 1.41188 & $5.64 \mathrm{E}-04$ & -8.3105 & - \\
\hline 15 & Cinnabarinic acid & $\mathrm{C}_{14} \mathrm{H}_{8} \mathrm{~N}_{2} \mathrm{O}_{6}$ & 300.0382 & 2.15 & 1.67405 & $2.14 \mathrm{E}-14$ & -8.3073 & - \\
\hline 16 & Spermidine & $\mathrm{C}_{7} \mathrm{H}_{19} \mathrm{~N}_{3}$ & 145.1579 & 2.07 & 1.54912 & $1.69 \mathrm{E}-05$ & -6.3363 & - \\
\hline
\end{tabular}

$\mathrm{RT}=$ retention time; VIP = variable important in the projection; $\mathrm{FC}=$ fold-change; FBM $=$ fermented brown milk; FM = fermented milk; $\mathrm{AUC}=$ area under the curve (only metabolites with AUC value $>0.8$ are shown). 


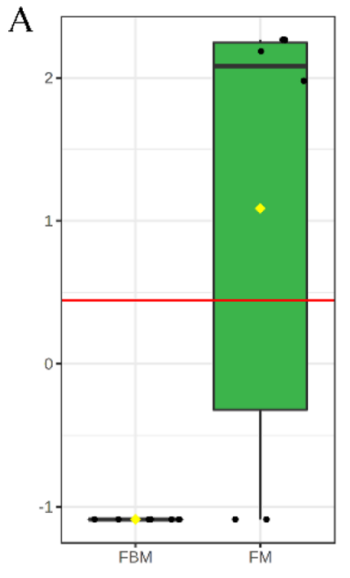

L-Lysine

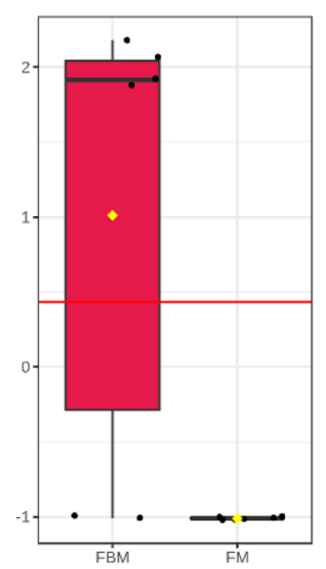

3-Hydroxybutanoic acid

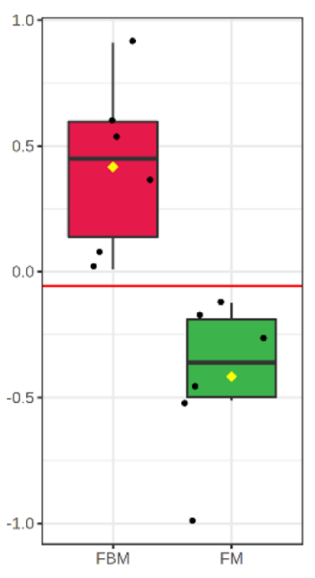

Methylglyoxal

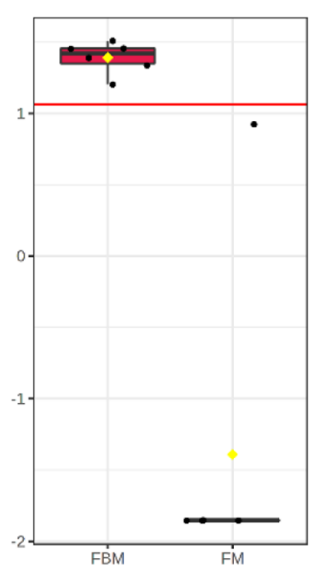

Glyoxal

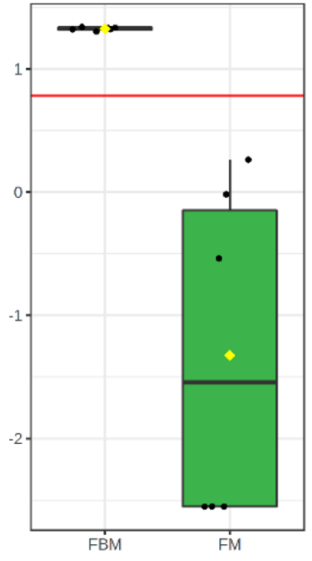

2,3-Pentanedione

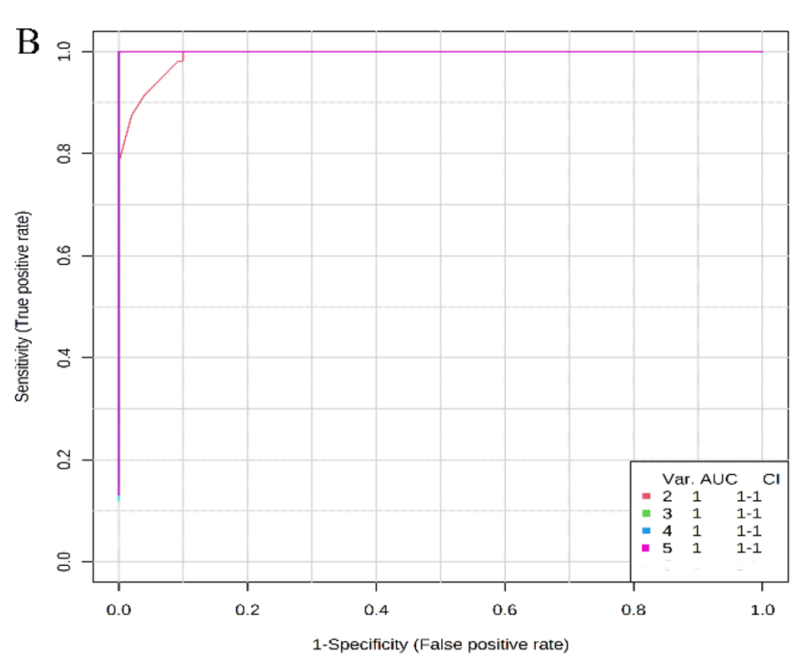

Figure 8. Five significant differential metabolites between fermented brown milk (FBM) and fermented milk (FM). (A) Differences in rela-

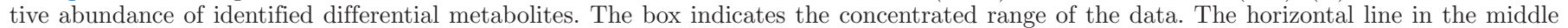

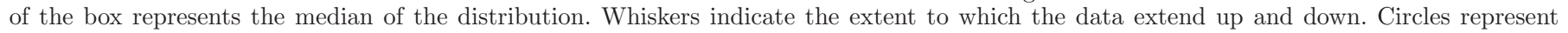

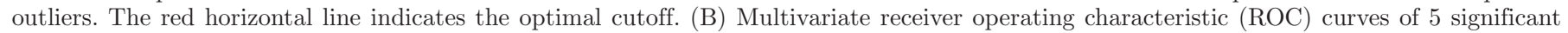
differential metabolites.

pyrimidine metabolism, arginine biosynthesis, butanoate metabolism, lysine biosynthesis, glycine, serine and threonine metabolism, arginine and proline metabolism, and taurine and hypotaurine metabolism. These pathways were mainly related to Maillard reaction-induced metabolic differences.

Amino Acids and Their Derivatives. Significant differences were found in the profile of AA-related compounds between the fermented brown milk and fermented milk metabolomes. Significantly less L-lysine, (R)-4'-phosphopantothenoyl-L-cysteine, and L-methionine S-oxide were detected in the fermented brown milk. As fermentation progressed, the starter culture $L$. bulgaricus used the milk proteins to sustain bacterial growth (Dong et al., 2017). Lysine reacts with reducing sugars during Maillard browning, which could explain the lower lysine level in the brown milk. We found that the L-lysine level in the fermented brown milk was much higher than that in brown milk, which could be caused by milk protein degradation by the starter bacteria during fermentation (Dong et al., 2017).

Some common changes in the AA profiles were observed in both fermented milk and fermented brown milk. For example, the levels of some free AA significantly increased after fermentation, such as L-isoleucine, L-phenylalanine, L-methionine, and L-valine, which were also reported previously in fermented milk produced by L. bulgaricus (Zhou et al., 2012). A high level of branched-chain AA in dairy products (e.g., L-isoleucine and L-threonine) act synergistically with calcium to ac- 
Peng et al.: FERMENTED BROWN MILK METABOLOMES
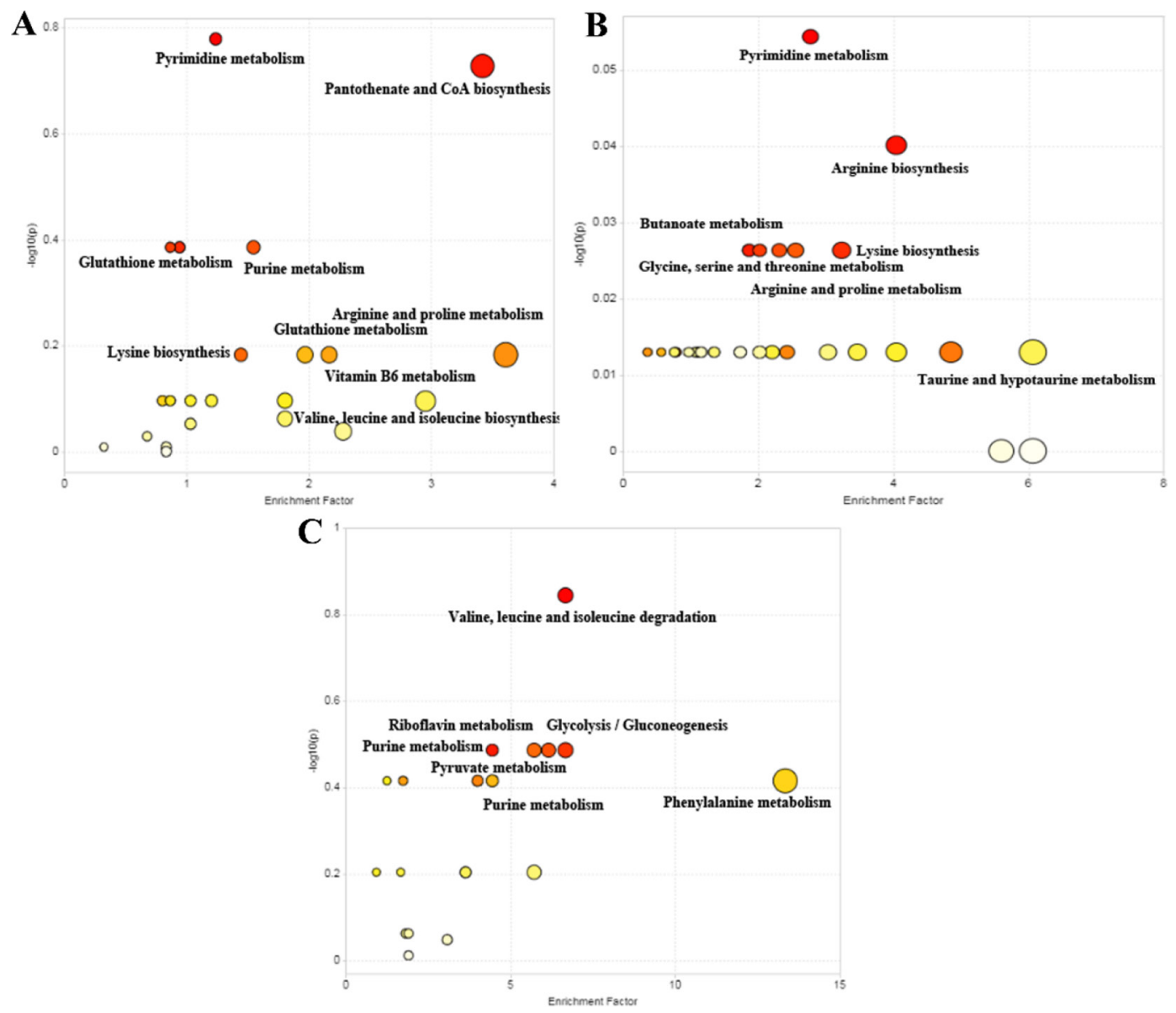

Figure 9. Mummichog pathway activity predicted between different milk sample pairs. (A) Fermented brown milk and brown milk; (B) fermented milk and milk; and (C) fermented brown milk and fermented milk.

celerate weight loss via adiposity attenuation (Zemel, 2004). Free AA also contribute to flavor by themselves or as precursors in the formation of other compounds (Hillmann and Hofmann, 2016). Thus, the rich variety of $\mathrm{AA}$ in the fermented brown milks not only serves as important nutrients and main taste contributors but also health-promoting bioactive components of these products.

Peptides. The content of peptides differed greatly between the fermented brown milk and fermented milk metabolomes. Fermented brown milk contained a much higher level (7,169.6 times higher) of glutathione than fermented milk. Glutathione is an active tripeptide formed by the condensation of glutamic acid, cysteine and glycine through peptide bonds, and it contains cysteine residues of sulfhydryl groups, making it the main intracellular antioxidant that serves important physiological functions in organisms (Zhu 2008). Maillard products were fermented by Lactobacillus gasseri $\mathrm{H} 10$ and Lactobacillus fermentum $\mathrm{H} 9$, and the overall preventive cardiovascular activity was improved. Also, they identified the production of small-molecule compounds from fermented Maillard reaction products (MRP) that probably had greater cardiovascular preventive effects than nonfermented (MRP) (Oh et al., 2014). These beneficial effects could be related to the antioxidation activity of glutathione in regulating cholesterol synthesis and metabolism (Oh et al., 2016). However, the biological function of the fermented brown milk produced in this study remains to be experimentally confirmed.

During fermentation, the starter bacteria ( $L$. bulgaricus) actively hydrolyzed the milk protein into polypeptides and AA (Zheng et al., 2012). A total of 5 identified differential metabolites were peptides in nature. The contents of 4 peptides, Ile-Lys-Gly-Pro, IlePro, Trp-Arg, and Pro-Trp were significantly higher in fermented milk compared with fermented brown milk $(P<0.05)$, whereas significantly $(P<0.05)$ less ValVla-Pro-Phe was detected in fermented milk. Three of the 4 peptides that were found to be increased, Ile-Pro, Pro-Trp, and Trp-Arg, are human dipeptidyl peptidase IV (hDPPIV) inhibitors that could reduce blood glu- 
cose concentration and ameliorate type 2 diabetes (Lan et al., 2015).

Fermentation of brown milk also increased the content of other peptides. Some of these peptides might contribute to the unique sensory quality of fermented brown milk (Li et al., 2020). Some other peptides identified in this study have been reported to influence the product taste (e.g., Gly-Gly-Pro and Gly-Gln-Ala-Arg were found to relate to sweetness and sourness, respectively; Li et al., 2020). Glu-Glu and Asp-Trp-Asp-Ser were found to be umami ( $\mathrm{Li}$ et al., 2020). The functional properties and beneficial effects of short peptides identified in the 2 types of fermented milks produced by ND02 need to be further investigated.

Aldehydes and Ketones. The levels of aldehyde and ketone metabolites were higher in fermented brown milk than in fermented milk. The levels of methylglyoxal, glyoxal, and 2,3-pentanedione were 87.80 times, 8.81 times and 27.61 times higher in the fermented brown milk than the fermented milk, respectively. Aldehydes are another important class of flavor substances in yogurt that have a relatively low flavor threshold. Previous studies have found that acetaldehyde, the main flavor substance in yogurt, is produced in fermentation by L. bulgaricus (Dan et al., 2012). Methylglyoxal and glyoxal could be produced during the Lactobacillus growth (O'Brien, 2009). Ketones are important flavor components of yogurt, which are produced by the oxidation and thermal degradation of polyunsaturated fatty acids, microbial metabolism, and AA degradation. 2,3-Pentanedione is associated with a creamy aroma and buttery aroma (Li et al., 2013b).

Lipid Metabolites. Lipid metabolites are another chemical group associated directly with the taste and flavor quality of fermented products. Bovine milk lipids mainly consist of triacylglycerols, diacylglycerols, monoacylglycerols, free fatty acids, phospholipids, and sterols. Four compounds related to lipid metabolism were identified between different milk metabolomes, including L-carnitine, lysophosphatidylcholine, glycerophosphocholine, and glyceric acid 1,3-biphosphate. The former 3 metabolites were initially present in milk in low or undetected levels, which increased gradually during the fermentation process. L-Carnitine and glycerophosphocholine were not detected in milk, but were detected both in fermented milk and fermented brown milk. Consistent results were reported in a previous study of dairy metabolomics that showed the content of L-carnitine increased during the fermentation process (Le Boucher et al., 2013). L-Carnitine is an AA metabolite that accelerates the transformation of fatty acids into energy, facilitating weight reduction; it is indispensable for glucose and fatty acid metabolism and hormonal regulation. Significantly more glycerophosphocholine was found in fermented milk than fermented brown milk. Significantly more lysophosphatidylcholine was detected in fermented milk than milk, which is an indicator of impaired glucose tolerance and type 2 diabetes. Lysophosphatidylcholine is known to activate natural killer cells, which could promote bacterial clearance and tumor rejection, as well as quell autoimmune disease pathology (Fox et al., 2009). Thus, it would be of interest to further investigate the role of these biomolecules in human health and their metabolism and interactions with LAB.

Organic Acids. Five significantly differential abundant organic acids or organic acid salts (i.e., cinnabarinic acid, butyryl-CoA, N6-acetyl-LL-2,6-diaminopimelate, $\mathrm{N}$-acetylmuramic acid 6-phosphate, and 3-hydroxybutanoic acid) were identified between fermented brown milk and fermented milk. The amount of N6-acetylLL-2,6-diaminopimelate in fermented brown milk was about 16-fold lower than in fermented milk. In contrast, the levels of $\mathrm{N}$-acetylmuramic acid 6-phosphate and 3-hydroxybutanoic acid were significantly higher in fermented brown milk $(P<0.05)$, and the amount of 3-hydroxybutanoic acid was even 274.51-fold higher than in fermented milk.

Organic acids are the primary products of carbohydrate catabolism of LAB. N6-Acetyl-LL-2,6-diaminopimelate is involved in the lysine synthesis pathway, and its decrease in fermented brown milk could be related to the loss of lysine in milk due to the Maillard reaction. On the other hand, fermentation by the starter bacteria could increase protein degradation and subsequently increase in fermented dairy products.

Only a small amount of lactic acid is normally present in milk. However, a large quantity of lactic acid is produced as nonvolatile flavor substance via carbohydrate metabolism of LAB during fermentation (Akalin et al., 2002; Izco et al., 2002). Apart from serving as a major flavor that contributes to the unique flavor of fermented milk, it also extends the shelf life of fermented milk, imparts acidity and a refreshing taste to the product, makes it easier to digest the protein, promotes gastrointestinal peristalsis, improves digestion, and improves absorption of calcium, phosphorus, iron, and other minerals present in fermented milk (Chen et al., 2007). Some other organic acids also serve both as natural flavor compounds and biopreservatives of fermented products. For example, benzoic acid is an antimicrobial compound that is a significant differential abundant metabolite detected in both fermented brown milk and fermented milk, which is likely produced by L. bulgaricus via transforming hippuric acid naturally present in milk (Park et al., 2017). 
Nucleotides and Related Metabolites. No significant differential nucleotide-related metabolites were found between the metabonomics of fermented brown milk and fermented milk. However, 5 differentially abundant nucleotides and related metabolites were detected between fermented milk and milk, including uric acid, xanthine, hyparaxanthine, uracil, and purine. All 5 compounds were significantly increased in fermented milk $(P<0.05)$. Uridine increased 861 -fold by the end of brown milk fermentation.

Nucleotides are an important class of nitrogen-containing compounds in the body. Milk nucleotides play a pivotal role in regulating the intestinal microflora, promoting growth and development, enhancing immunity, and improving lipid metabolism. Lactobacillus bulgaricus can use purine as a carbon, nitrogen, and energy source for growth. In the process of purine decomposition, adenine is decomposed by adenine dehydrogenase to form hypoxanthine, which is further converted to xanthine by hypoxanthine oxidase. In addition, guanine can also generate xanthine via catalysis by guanine dehydrogenase. Finally, xanthine is catalyzed to form uric acid by the action of xanthine oxidase (Suzuki et al., 1986). The increase in the levels of these nucleotide-related compounds in fermented milk and fermented brown milk can thus be explained by the active metabolism of the starter bacteria in the fermentation process.

Vitamins. No significant differential vitamin metabolites were found between the metabolomes of fermented brown milk and fermented milk. However, the present study found an 8.78-fold enrichment of nicotinamide- $N$-oxide in fermented milk compared with milk. Nicotinamide- $N$-oxide is an intermediate product of vitamin B12 synthesis by LAB (Moore and Warren, 2012). An in vitro screen found that 8 of 16 tested LAB strains, including one Lactobacillus, had the ability to produce vitamin B12 (Masuda et al., 2012). On the other hand, less riboflavin was present in the fermented milk compared with milk, which could be explained by the intrinsic lack of some other vitamin biosynthetic pathways in LAB, making these bacteria nutritionally fastidious. Thus, they had to utilize certain vitamins in milk for growth, such as riboflavin and pyridoxal (Le Boucher et al., 2013).

The level of vitamin A increased significantly in both kinds of fermented milk (349-fold increase in fermented milk compared with milk; 231-fold increase in fermented brown milk compared with brown milk; $P<0.05$ in both cases), suggesting that the L. bulgaricus ND02 strain used in this study was potentially producing vitamin A. This is supported by Panfili et al. (1998), who observed extensive retinol isomerization in cheese. However, only little is known about the metabolic path- ways of vitamin A synthesis by L. bulgaricus, which is worthy of investigation.

\section{CONCLUSIONS}

In this work, we used a metabolomic approach to analyze chemical and nutritional differences among 4 products, milk, brown milk, fermented milk, and fermented brown milk. Significant differences were detected in the abundances of various groups of metabolites, including peptides, AA, aldehydes, ketones, organic acids, vitamins, and nucleosides. Our data showed that the Maillard browning reaction significantly increased a wide spectrum of flavor compounds such as short peptides, aldehydes, ketones, organic acids, sulfur, and furan compounds. They might together contribute to the unique flavor of brown milk. However, Maillard browning also resulted in significant increase in carboxymethyllysine, which might have negative health impacts. On the other hand, fermenting brown milk with the starter culture, L. bulgaricus ND02, effectively countered such effect. Finally, 6 differential abundant metabolites were identified between fermented milk and fermented brown milk metabolomes, including Llysine, methylglyoxal, glyoxal, 2,3-pentanedione, and 3-hydroxybutanoic acid. Some of these biomolecules might be account for the different nutritional qualities between these 2 types of fermented products.

\section{ACKNOWLEDGMENTS}

This work was supported by the National Natural Science Foundation of China, Beijing (grant no. 31771954), National Natural Science Foundation of China, Beijing (grant no. 31922071), Inner Mongolia Autonomous Region Science and Technology Achievement Transformation Project, Hohhot (2019CG027), and Natural Science Foundation of Inner Mongolia Autonomous Region of China, Hohhot (2021ZD08). Jiangying Peng and Wenyi Zhang participated in project design; Tiansong Sun supervised the project; Jiangying Peng and Liqing Ma performed the experiments; Jiangying Peng analyzed and interpreted the data and drafted the manuscript. Lai-Yu Kwok extensively revised the manuscript. All authors have read and approved the content of the manuscript. The authors have not stated any conflicts of interest.

\section{REFERENCES}

Aalaei, K., M. Rayner, and I. Sjöholm. 2019. Chemical methods and techniques to monitor early Maillard reaction in milk products; A review. Crit. Rev. Food Sci. Nutr. 59:1829-1839. https://doi.org/ 10.1080/10408398.2018.1431202. 
Akalin, A. S., S. Gönç, and Y. Akba. 2002. Variation in organic acids content during ripening of pickled white cheese. J. Dairy Sci. 85:1670-1676. https://doi.org/10.3168/jds.S0022-0302(02)74239 -2 .

Ashoor, S. H., and J. B. Zent. 1984. Maillard browning of common amino acids and sugars. J. Food Sci. 49:1206-1207. https://doi .org/10.1111/j.1365-2621.1984.tb10432.x.

Burgos-Rubio, C. N., R. Okos, and P. C. Wankat. 2000. Kinetic study of the conversion of different substrates to lactic acid using Lactobacillus bulgaricus. Biotechnol. Prog. 16:305-314. https://doi.org/ 10.1021/bp000022p.

Buttriss, J. 1997. Nutritional properties of fermented milk products. Int. J. Dairy Technol. 50:21-27. https://doi.org/10.1111/j.1471 -0307.1997.tb01731.x.

Chen, Y. F., J. C. Wang, Z. Y. Yun, and H. P. Zhang. 2007. Study on organic acids in traditionally ferm ented milk by HPLC. Dairy Industry. 35:54-58. https://doi.org/10.3969/j.issn.1001-2230.2007 .01 .014 .

Dan, T., Q. H. Bao, H. B. L. Meng, J. G. Wang, and H. P. Zhang. 2012. Gene regulation to increase acetaldehyde and diacetyl production by lactic acid bacteria. Food Sci. Technol. 37:75-79.

Dan, T., H. Y. Chen, T. Li, J. L. Tian, W. Y. Ren, H. P. Zhang, and T. S. Sun. 2019. Influence of Lactobacillus plantarum P-8 on fermented milk flavor and storage stability. Front. Microbiol. 9:3133. https://doi.org/10.3389/fmicb.2018.03133.

Do Carmo, A. P., D. Da Silva, M. De Oliveira, A. Borges, A. De Carvalho, and C. De Moraes. 2011. Genes involved in protein metabolism of the probiotic lactic acid bacterium Lactobacillus delbrueckii UFV H2b20. Benef. Microbes 2:209-220. https://doi.org/10.3920/ BM2011.0025.

Dong, H., Y. Yu, J. Yan, and Y. Jin. 2017. Tracking casein phosphopeptides during fermentation by high performance liquid chromatography-tandem mass spectrometry. Se Pu 35:587-593. https:// doi.org/10.3724/SP.J.1123.2017.03012.

Fox, L. M., D. G. Cox, J. L. Lockridge, X. Wang, X. Chen, L. Scharf, D. L. Trott, R. M. Ndonye, N. Veerapen, G. S. Besra, A. R. Howell, M. E. Cook, E. J. Adams, W. H. Hildebrand, and J. E. Gumperz. 2009. Recognition of lyso-phospholipids by human natural killer T lymphocytes. PLoS Biol. 7:e1000228. https://doi.org/10.1371/ journal.pbio.1000228.

Gu, F., J. M. Kim, K. Hayat, S. Xia, B. Feng, and X. Zhang. 2009. Characteristics and antioxidant activity of ultrafiltrated Maillard reaction products from a casein-glucose model system. Food Chem. 117:48-54. https://doi.org/10.1016/j.foodchem.2009.03.074.

Han, Z., J. Gao, J. Li, Y. Zhang, Y. Yang, and S. Wang. 2019. Mitigation of 3-deoxyglucosone and 5-hydroxymethylfurfural in brown fermented milk via an alternative browning process based on the hydrolysis of endogenous lactose. Food Funct. 10:2022-2029. https: //doi.org/10.1039/C8FO02023J.

Hillmann, H., and T. Hofmann. 2016. Quantitation of key tastants and re-engineering the taste of Parmesan cheese. J. Agric. Food Chem. 64:1794-1805. https://doi.org/10.1021/acs.jafc.6b00112.

Izco, J. M., M. Tormo, and R. Jiménez-Flores. 2002. Rapid simultaneous determination of organic acids, free amino acids, and lactose in cheese by capillary electrophoresis. J. Dairy Sci. 85:2122-2129. https://doi.org/10.3168/jds.S0022-0302(02)74290-2.

Lan, V. T. T., K. Ito, M. Ohno, T. Motoyama, S. Ito, and Y. Kawarasaki. 2015. Analyzing a dipeptide library to identify human dipeptidyl peptidase IV inhibitor. Food Chem. 175:66-73. https://doi .org/10.1016/j.foodchem.2014.11.131

Le Boucher, C., F. Courant, S. Jeanson, S. Chereau, M. B. Maillard, A. L. Royer, A. Thierry, G. Dervilly-Pinel, B. L. Bizec, and S. Lortal. 2013. First mass spectrometry metabolic fingerprinting of bacterial metabolism in a model cheese. Food Chem. 141:1032-1040. https://doi.org/10.1016/j.foodchem.2013.03.094.

Li, D., Y. Zheng, L. Y. Kwok, W. Y. Zhang, and T. S. Sun. 2020. Metabolic footprinting revealed key biochemical changes in a brown fermented milk product using Streptococcus thermophilus. J. Dairy Sci. 103:2128-2138. https://doi.org/10.3168/jds.2019-16881.

Li, S., Y. Park, S. Duraisingham, F. H. Strobel, N. Khan, Q. A. Soltow, D. P. Jones, and B. Pulendran. 2013a. Predicting network activity from high throughput metabolomics. PLOS Comput. Biol. 9:e1003123. https://doi.org/10.1371/journal.pcbi.1003123.

Li, Y. H., L. Zhang, and W. Wang. 2013b. Heat-induced changes in volatiles of milk and effects of thermal processing on microbial microbial metabolism of yogurt. J. Food Biochem. 37:409-417. https: //doi.org/10.1111/j.1745-4514.2011.00642.x.

Liu, X., M. Zhang, X. Cheng, X. Liu, H. Sun, Z. Guo, J. Li, X. Tang, Z. Wang, W. Sun, Y. Zhang, and Z. Ji. 2020. LC-MS-based plasma metabolomics and lipidomics analyses for differential diagnosis of bladder cancer and renal cell carcinoma. Front. Oncol. 10:717-14. https://doi.org/10.3389/fonc.2020.00717.

Masuda, M., M. Ide, H. Utsumi, T. Niiro, Y. Shimamura, and M. Murata. 2012. Production potency of folate, vitamin b-12, and thiamine by lactic acid bacteria isolated from Japanese pickles. Biosci. Biotechnol. Biochem. 76:2061-2067. https://doi.org/10 $.1271 /$ bbb.120414.

Moore, S. J., and M. Warren. 2012. The anaerobic biosynthesis of vitamin B12. Biochem. Soc. Trans. 40:581-586. https://doi.org/ 10.1042/BST20120066

Mottram. D. S., B. L. Wedzicha, and A. T. Dodson. 2002. Acrylamide is formed in the Maillard reaction. Nature 419:448-449. https:// doi.org/10.1038/419448a.

Ni, D., N. Li, H. B. Zhang, and G. W. Ma. 2017. Development of brown drinking yoghurt. Zhongguo Rupin Gongye 4:56-58.

O'Brien, J. 2009. Non-enzymatic degradation pathways of lactose and their significance in dairy products. Pages 231-294 in Advanced Dairy Chemistry. P. McSweeney and P. Fox, ed. Springer. https:// doi.org/https://doi.org/10.1007/978-0-387-84865-5_7.

Oh, N. S., J. H. Koh, M. R. Park, Y. Kim, and S. H. Kim. 2016. Short communication: Hypolipidemic and antiinflammatory effects of fermented Maillard reaction products by Lactobacillus fermentum H9 in an animal model. J. Dairy Sci. 99:9415-9423. https://doi .org/10.3168/jds.2016-11286.

Oh, N. S., H. S. Kwon, H. A. Lee, J. Y. Joung, J. Y. Lee, K. B. Lee, Y. K. Shin, S. C. Baick, M. R. Park, Y. Kim, K. W. Lee, and S. H. Kim. 2014. Preventive effect of fermented Maillard reaction products from milk proteins in cardiovascular health. J. Dairy Sci. 97:3300-3313. https://doi.org/10.3168/jds.2013-7728.

Panfili, G., P. Manzi, and L. Pizzoferrato. 1998. Influence of thermal and other manufacturing stresses on retinol isomerization in milk and dairy products. J. Dairy Res. 65:253-260. https://doi.org/10 .1017/S0022029997002811.

Park, S. Y., M. Y. Yoo, H. D. Paik, and S. D. Lim. 2017. Production of benzoic acid as a natural compound in fermented skim milk using commercial cheese starter. J. Dairy Sci. 100:4269-4275. https:// doi.org/10.3168/jds.2016-12399.

Rannou, C., D. Laroque, E. Renault, C. Prost, and T. Sérot. 2016. Mitigation strategies of acrylamide, furans, heterocyclic amines and browning during the Maillard reaction in foods. Food Res. Int. 90:154-176. https://doi.org/10.1016/j.foodres.2016.10.037.

Roberts, L. D., A. L. Souza, R. E. Gerszten, and C. B. Clish. 2012. Targeted metabolomics. Curr. Protoc. Mol. Biol. 98:30.2.1-30.2.24. https://doi.org/10.1002/0471142727.mb3002s98.

Rochfort, S. 2005. Metabolomics reviewed: A new "omics" platform technology for systems biology and implications for natural products research. J. Nat. Prod. 68:1813-1820. https://doi.org/10 $.1021 / \mathrm{np} 050255 \mathrm{w}$.

Shida, K., T. Sato, R. Iizuka, R. Hoshi, O. Watanabe, T. Igarashi, K. Miyazaki, M. Nanno, and F. Ishikawa. 2017. Daily intake of fermented milk with Lactobacillus caseistrain Shirota reduces the incidence and duration of upper respiratory tract infections in healthy middle-aged office workers. Eur. J. Nutr. 56:45-53. https: //doi.org/10.1007/s00394-015-1056-1.

Sudun, W., K. Wulijideligen, M. Arakawa, Miyamoto, and T. Miyamoto. 2013. Interaction between lactic acid bacteria and yeasts in airag, an alcoholic fermented milk. Anim. Sci. J. 84:66-74. https:/ /doi.org/10.1111/j.1740-0929.2012.01035.x.

Sun, Z., W. J. Liu, W. Gao, M. Yang, J. C. Zhang, L. Wu, J. G. Wang, B. L. Menghe, T. S. Sun, and H. P. Zhang. 2010. Identification and characterization of the dominant lactic acid bacteria from kurut: 
The naturally fermented yak milk in Qinghai, China. J. Gen. Appl. Microbiol. 56:1-10. https://doi.org/10.2323/jgam.56.1.

Suzuki, I., S. Kato, T. Kitada, N. Yano, and T. Morichi. 1986. Growth of Lactobacillus bulgaricus in milk. 2. Characteristics of purine nucleotides, pyrimidine nucleotides, and nucleic acid synthesis. J. Dairy Sci. 69:971-978. https://doi.org/10.3168/jds.S0022 -0302(86)80490-8.

Worley, B., and R. Powers. 2016. PCA as a practical indicator of OPLS-DA model reliability. Curr. Metabolomics 4:97-103. https:/ /doi.org/10.2174/2213235X04666160613122429.

Xu, Z. Y., Y. Wu, B. H. Guo, L. H. Zhou, Y. Y. Wang, and L. Z. Ai. 2010. Research and preparation of a brown milk drink with probiotic. Sci. Technol. Food Industry. 31:242-244. https://doi.org/10 .13386/j.issn1002-0306.2010.08.057.

Yu, A. N., Z. W. Tan, and F. S. Wang. 2012. Mechanism of formation of sulphur aroma compounds from l-ascorbic acid and l-cysteine during the Maillard reaction. Food Chem. 132:1316-1323. https:/ /doi.org/10.1016/j.foodchem.2011.11.111.

Zemel, M. B. 2004. Role of calcium and dairy products in energy partitioning and weight management. Am. J. Clin. Nutr. 79:907S-912S. https://doi.org/10.1093/ajcn/79.5.907S.

Zha, M., K. Li, W. Zhang, Z. Sun, L.-Y. Kwok, B. Menghe, and Y. Chen. 2021. Untargeted mass spectrometry-based metabolomics approach unveils molecular changes in milk fermented by Lactobacillus plantarum P9. Lebensm. Wiss. Technol. 140:110759. https:/ /doi.org/10.1016/j.lwt.2020.110759.

Zheng, H., E. Liu, P. Hao, T. Konno, M. Oda, and Z.-S. Ji. 2012. In silico analysis of amino acid biosynthesis and proteolysis in Lactobacillus delbrueckii ssp. bulgaricus 2038 and the implications for bovine milk fermentation. Biotechnol. Lett. 34:1545-1551. https: /doi.org/10.1007/s10529-012-1006-4.

Zhou, Wei, F. Yu, and L. J. Chen. 2012. Study on variations of essential amino acid in fermented milk during fermentation and cold storage. Zhongguo Shiwu yu Yingyang 18:26-28.

Zhu, Y. Q. 2008. Research progress of glutathione production by fermentation. Zhongguo Fajiao Keji Tongxun. 2:22-26.

\section{ORCIDS}

Jiangying Peng ๑ https://orcid.org/0000-0001-7140-5740

Lai-Yu Kwok @ https://orcid.org/0000-0001-8791-1269

Wenyi Zhang @ https://orcid.org/0000-0001-5530-4210 